Classification

Physics Abstracts

$61.70 \mathrm{~N}-91.70 \mathrm{Y}-62.20$

\title{
Geometrical speciality and special properties of grain boundaries
}

\author{
L. Priester \\ Laboratoire de Métallurgie Structurale, U.A. CNRS 1107, Université Paris Sud, 91405 Orsay, France
}

(Reçu le 28 novembre 1988, révisé le 5 janvier 1989, accepté le 12 janvier 1989)

\begin{abstract}
Résumé. - De tout temps, les utilisateurs des matériaux ont tenté de contrôler leurs microstructures dans le but de leur conférer des propriétés spécifiques pour un emploi donné. C'est de ce point de vue que cet article de synthèse considère les joints de grains, éléments fondamentaux de la microstructure polycristalline, avec deux objectifs : a) tenter d'élucider les relations entre la cristallographie d'un joint de grains, qui détermine sa structure, et différentes propriétés de ce joint ; b) établir la distribution des joints de grains dans un polycristal selon leurs caractéristiques géométriques afin de comprendre leur contribution aux propriétés de l'ensemble polycristallin.
\end{abstract}

\begin{abstract}
It has long been a major preoccupation of materials users, whether implied or explicit, to tailor microstructures in order to adapt specific properties for a given application. In this context, the principal aim of the present review article is to consider the fundamental elements of polycrystal microstructures, i.e. the grain boundaries. The paper discusses current understanding of two important aspects : a) the possible relationships between the geometry of a grain boundary, which determines its structure, and its various properties ; b) the distribution of geometrical characteristics, which for real boundaries can only be approached experimentally, in an endeavour to determine the intergranular contribution to the overall properties of a polycrystal.
\end{abstract}

\section{Introduction.}

The question of the correspondance between the geometrical speciality $\left({ }^{1}\right)$ of grain boundaries and special intergranular properties has become extremely topical for two essential reasons :

- the increasing ambiguity between the « geometry-structure " relationship and the assertion of the continuity and multiplicity of intergranular structures $[1-6]$;

- the general acceptance by crystal interface specialists of the "grain boundary design » concept advanced some years ago by Watanabe [7].

The clearest evidence for the growing interest in this subject is the rise in the number of papers and

(1) The geometry is defined here by the macroscopic crystallographic parameters : misorientation $(\theta,[u v w])$ and grain boundary plane $(h k \ell)$ that generally control most macroscopic properties. Nevertheless, care must be taken concerning grain boundary diffusion measurements that may be affected by the rigid body translation microscopic parameters. international meetings of which it is the central theme.

The aim of the present review article is to describe the present situation of the «structure-properties » relationship by considering :

- the historical development of the concept of « speciality » (Sect. 2);

- available evidence concerning the relation between geometry and properties (Sect. 3) ;

- present prospects for tailoring the properties of polycrystalline materials via the control of grain boundaries (Sect. 4). In order to do this, a first requirement is to determine the distribution of boundaries according to their geometries (designated « grain boundary texture »).

\section{The concept of geometrical speciality.}

Implicit in the term "geometrical speciality " is the assumption that specific values of misorientation and/or certain grain boundary planes can lead to particular intergranular structures. This possibility will be discussed below. 
2.1 SPECIALITY BASED ON THE NOTION OF COINCIDENCE. - In early models, in which grain boundaries were considered as amorphous layers [8], the idea of speciality is totally absent. This notion was first introduced by Read and Shockley in their description of low-angle boundaries $\left(\theta<15^{\circ}\right)$ [9]. Based on Friedel's coincidence concept [10], Bollmann then developed a geometrical approach, using two mathematical tools, the zero-lattice and the DSC-lattice (displacement shift complete), in which any grain boundary can be formally considered in terms of periodic arrangements of intrinsic dislocations [11]. This led to a basic contradiction, which has never been resolved, between the theoretical continuity of intergranular order and the necessity to define a «physically significant » order corresponding to some criterion of speciality. The first transmission electron microscope (TEM) observations on bicrystals of controlled misorientation favoured a physical order linked to the visualization of intrinsic dislocations [12-14]. For symmetrical pure twist or pure tilt boundaries, critical angular deviations either from a single crystal $\left(\theta \leqslant 20^{\circ}\right)$ or from a coincidence misorientation with $\Sigma \leqslant 25$ are then defined as speciality criteria, provided that intrinsic dislocation networks are visible in the TEM. $\Sigma$ is the inverse density of coincidence sites. The critical angular deviation $\Delta \theta_{c}$ increases with decreasing $\Sigma$.

Later, the existence of primary dislocation networks in grain boundaries with misorientations up to $45^{\circ}$ were revealed indirectly by $\mathrm{X}$-ray diffraction $[15$, 16], providing the first evidence that the geometrical continuity of the O-lattice is physically relevant. At about the same time, it was found that simple periodic arrays of dislocations could be observed in grain boundaries of any misorientation provided that the axis of the latter corresponded to a close-packed direction of the crystal lattice. In f.c.c. metals, this geometry leads to good matching across the boundary of the most closely-packed planes $\{111\}$, $\{200\}$ or $\{220\}$ [17]. This « matching-plane » model was later described as being an extreme case of coincidence, obtained when $\Sigma$ tends to infinity [18], a coincidence axis direction being preserved in the boundary plane [19].

At this stage, three types of symmetrical boundaries were considered to have «special " characteristics :

- low angle boundaries : $\theta \leqslant 20^{\circ}$;

- Coincidence Site Lattice $\left({ }^{2}\right)$ (CSL) boundaries :

$$
\Sigma \leqslant 25, \quad \Delta \theta=\theta_{\exp }-\theta_{\mathrm{CSL}} \leqslant \Delta \theta_{\mathrm{c}}
$$

(2) The Coincidence Lattice (CL previously called CSL) is obtained by the intersection I of the translation subgroups $\mathrm{T}^{\mathrm{I}}$ and $\mathrm{T}^{\mathrm{II}}$ of the two crystals $: \mathrm{I}=\mathrm{T}^{\mathrm{I}} \cap \mathrm{T}^{\mathrm{II}}$.
- Coincidence Axis Direction (CAD) boundaries :

$$
\Sigma \rightarrow \infty, \quad[u v w]_{\mathrm{fcc}}=\langle 100\rangle,\langle 110\rangle \text { or }\langle 111\rangle .
$$

A more suitable terminology is now generally adopted for CSL and CAD boundaries, respectively three-dimensional and one-dimensional coincidence grain boundaries.

In all cases, the intrinsic dislocations can only be visualized for a particular orientation of the grain boundary plane, which is not frequently encountered in real polycrystals.

In any case, a speciality criterion which is related to experimental techniques is obviously not satisfactory. Nevertheless, based on the principle that, even in the absence of experimental evidence, three dimensional coincidence can still occur between two crystals whose relative orientations are close to a CSL conditions $\left({ }^{3}\right)$, a number of more general criteria have been defined which are particularly useful for grain boundaries in polycrystals. All these arbitrary criteria are based on the probability that any grain boundary will possess a periodic structure, provided that its misorientation does not exceed the critical deviation $\Delta \theta_{c}$ from the coincidence orientation, given by

$$
\Delta \theta_{\mathrm{c}}=b / p
$$

where $b$ is a possible Burgers vector of the intergranular dislocations :

$$
b=b_{\mathrm{DSC}}=\Sigma^{-1 / n}
$$

and $p$ is a possible periodicity in the boundary plane :

$$
p=\Sigma^{-1 / m} \text {. }
$$

Different values have been attributed to $m$ and $n$, giving rise to more or less restrictive criteria [24-26]. The most frequently employed criterion is that due to Brandon:

$$
\Delta \theta_{c}=15^{\circ} \Sigma^{-1 / 2}
$$

$15^{\circ}$ being the limiting misorientation for low-angle boundaries.

(3) This statement is only true for materials with cubic symmetry. The concept of exact three dimensional coincidence has very limited applications in the case of structures with lower symmetry, for which it only exists for a particular axis of rotation, such as the sixfold axis in hexagonal systems or the threefold axis in rhombohedral crystals. In most cases, only a near coincidence relation can be obtained by the rotation of one grain with respect to the other. An additional deformation is then required to superimpose the multiple unit cells of the two crystals, chosen for similarity of shape and size, so as to obtain a coincidence lattice [20-23]. 

if :

A boundary is therefore considered to be special

$$
\Delta \theta_{\exp } \leqslant \Delta \theta_{\mathrm{c}}
$$

Whether they involve either real or virtual intrinsic dislocations, the above criteria are implicitly based on the principle that a grain boundary will maintain a coincidence structure because it is energetically favourable. However, both measurements and calculations show that an implied simple relationship between the coincidence index $\Sigma$ and boundary

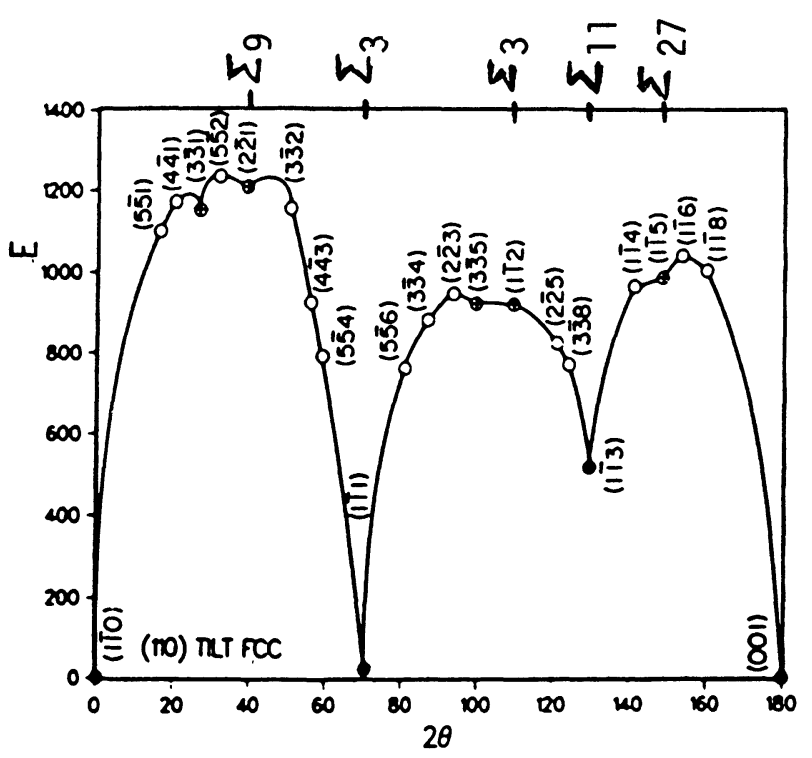

a)

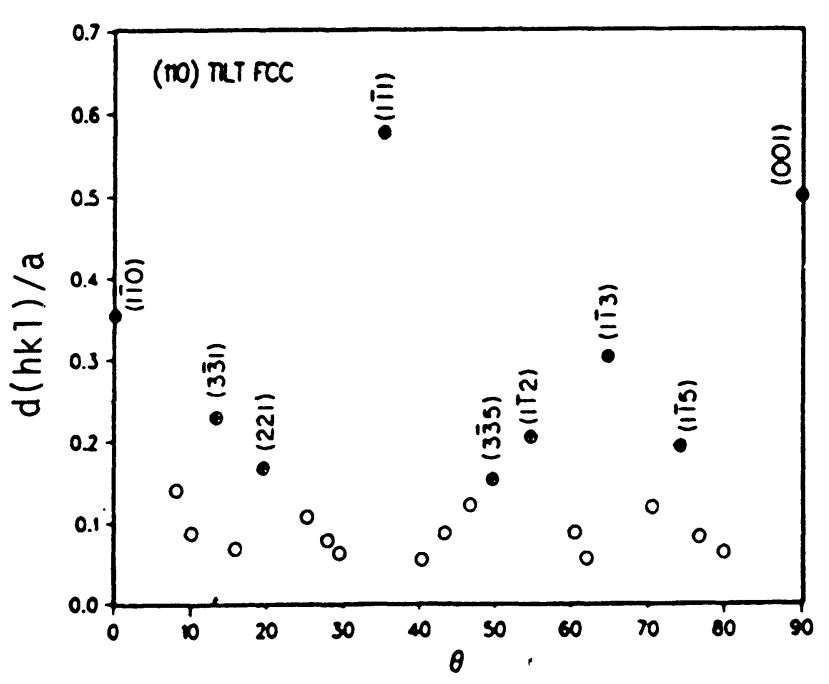

b)

Fig. 1. - a) Relaxed Energies of $\langle 110\rangle$ symmetrical tilt grain boundaries in $\mathrm{Cu}$ determined by means of the spline potential versus tilt angle $2 \theta$ [27]. b) The corresponding interplanar lattice spacings parallel to the grain boundary plane. Note that : Primary cusps are associated with high spacings $(\bullet)$ and secondary cusps with intermediate spacings $(\oplus)$. energy does not exist. Not only does a low value of $\Sigma$ not necessarily correspond to a minimum energy, but the orientation of the boundary plane also has a far from negligible influence (Fig. 1) [27].

An atomistic representation of grain boundaries thus appeared to offer a more fruitful approach in the quest for an improved criterion of speciality.

\subsection{SPECIALITY BASED ON THE STRUCTURAL UNIT} MODEL. - Grain boundary energy calculations have shown that the atoms in the intergranular region adopt a limited number of polyhedral configurations, and any boundary may be described as an arrangement of these polyhedra or «structural units » [28-30]. Only those grain boundaries that are composed of regular arrays of a single type of structural unit, known as «delimiting boundaries", possess a relatively low energy. The structural unit may either be "primitive", in which case the boundaries are said to be "favoured", or may be composed of different, slightly distorted elements, forming a "multiple unit reference structure » [30]. Any special «CSL » boundary can be described as a simple combination of the basic units associated with two favoured boundaries with misorientations on either side of the one considered (Fig. 2b) [29]. The sequence becomes more complex for "general » grain boundaries, i.e. with a high $\Sigma$ value, and can involve arrangements such as : AAA B AAA B... (Fig. 2c).

The geometrical and atomistic descriptions were for a long time considered independently, but were combined in a single model, designated SU/GBD (Structural Unit/Grain Boundary Dislocation), when it was found that the periodicity of the minority units was identical to that of the secondary dislocations [31, 32]. Moreover, the continuity of the intergranular order was confirmed. Thus, while the atomistic approach led to a better understanding of grain boundary structure, it did not solve the problem of defining a criterion of speciality. Indeed, it might even be argued that, being ordered, most, if not all, boundaries are special. In practice, two possible attitudes can be adopted :

a) only favoured boundaries are considered to be really special ;

b) an arbitrary limit is selected for the degree of organization of the structural units (and therefore of the dislocations); this procedure is analogous to the definition of a critical angular deviation discussed above (cf. Sect. 2).

Whatever the solution chosen, the use of the SU/GBD model to define a speciality criterion was made questionable by the discovery that a single boundary can have a multiplicity of structures. On the one hand, a given delimiting boundary can be described by different basic units of similar energy $[4,30]$, so that the various combinations give rise to 


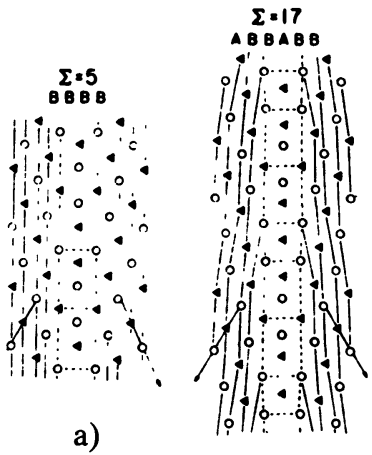

b)

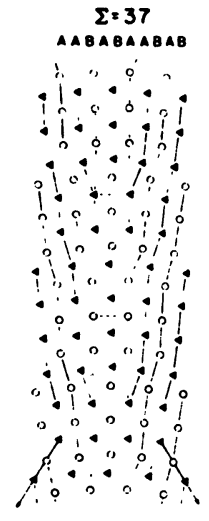

c)
Fig. 2. - Core structures of relaxed [001] symmetrical tilt boundaries in $\mathrm{Cu}$ [29]. $\Sigma=1$ (d) and $\Sigma=5$ (a) are the delimiting « favoured " grain boundaries, $A$ and $B$ being the respective structural units. The grain boundary $\Sigma=37$ is constituted by an arrangement of units A and B (c) a little more complex than the sequence of these units in $\Sigma=17$ (b). a plurality of possible intergranular structures (Fig. 3). On the other hand, any grain boundary can be described by choosing two different delimiting boundaries with misorientations more or less close to the value considered. The large number of configurations which result can be considered in terms of a hierarchy of dislocation arrays (of order 1 , $2,3 \ldots$ and so on) [33]. The higher order GBD's are then considered as disturbances in the lower order arrays, (Tab. I, Fig. 4).

The physical observation of these various arrangements depends on the conditions under which the boundary structure is examined. Moreover, certain experimentally determined periodic configurations (e.g. from X-ray or electron diffraction patterns) do not always correspond to energetically favourable structures [6]. This situation leads back to the dichotomy between theoretical and physical order.

Thus, the SU/GBD model fails to establish a straightforward relationship between energy and intergranular structure, even in the simple case of

Table I. - List of delimiting boundaries used in various hierarchical members of the SU/GBD description of [001] symmetrical tilt boundaries. $n$ represents the order of the member in the hierarchy.

\begin{tabular}{|c|c|c|c|c|c|c|c|c|}
\hline \multirow{2}{*}{$\frac{n}{1}$} & \multicolumn{8}{|c|}{ Delimiting boundaries } \\
\hline & $\Sigma 1(110)$ & $\Sigma 1(100)$ & & & & & & \\
\hline 2 & $\Sigma 1(110)$ & $55(210)$ & $\Sigma 1(100)$ & & & & & \\
\hline 3 & $\Sigma 1(110)$ & $\Sigma 5(210)$ & $\Sigma 5(310)$ & $\Sigma 1(100)$ & & & & \\
\hline 4 & $\Sigma 1(110)$ & $\Sigma 13(320)$ & $55(210)$ & $55(310)$ & $\Sigma 1(100)$ & & & \\
\hline 5 & $\Sigma 1(110)$ & $\Sigma 13(320)$ & $\Sigma 5(210)$ & $55(310)$ & $\Sigma 17(410)$ & $\Sigma 1(100)$ & & \\
\hline 6 & $\Sigma 1(110)$ & $\Sigma 13(320)$ & $\Sigma 5(210)$ & $\Sigma 5(310)$ & $\Sigma 17(410)$ & $\Sigma 13(510)$ & $\Sigma 1(100)$ & \\
\hline 7 & $\Sigma 1(110)$ & $\Sigma 13(320)$ & $\Sigma 17(530)$ & $55(210)$ & $25(310)$ & $\Sigma 17(410)$ & $\Sigma 13(510)$ & $\Sigma 1(100)$ \\
\hline$\vdots$ & $\vdots$ & & & & & & & \\
\hline
\end{tabular}

symmetrical grain boundaries. It therefore appears all the more improbable that general grain boundary properties (including their energy) will be able to be predicted from cristallographic parameters in the near future.

Due to the limitations of the preceding models, together with the necessity of defining a less sophisticated criterion of speciality, applicable to a random boundary in a polycrystalline material, other methods were employed in an attempt to predict boundary character, or simply to establish guidelines for the investigation of intergranular structure.

\subsection{SPECIALITY BASED ON THE ORIENTATION OF} THE BOUNDARY PLANE. - Wolf [34] was the first to suggest that the orientation of the grain boundary plane might constitute a useful parameter for establishing a new criterion of speciality. His analysis is based in particular on two observations :
- when the energy of symmetrical boundaries is plotted as a function of misorientation about an axis $[u v w]$, minima, or «cusps » are found to occur when the boundary is parallel to a close-packed crystal plane (Fig. 1) ;

- a small deviation from a cusp misorientation leads to a rapid increase in energy for a symmetrical tilt boundary, the plane of the latter being modified. On the contrary, the energy varies very little in the case of a twist boundary.

This implies that the planar density of coincidence sites $\sigma\left({ }^{4}\right)$ is not the only factor which controls the interfacial energy and indicates the importance of the atomic density in the boundary plane, which is

$\left({ }^{4}\right) \sigma$ is implicitly taken into account in the $\Sigma$ criterion of the CSL model, since the boundary plane is never considered to be randomly oriented (cf. Sect. 2.1). 
a)

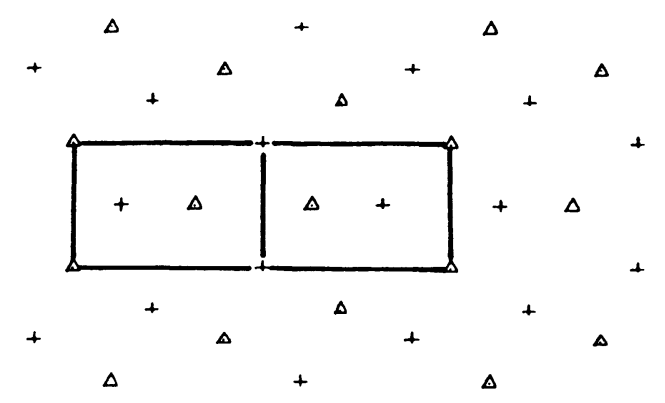

b)

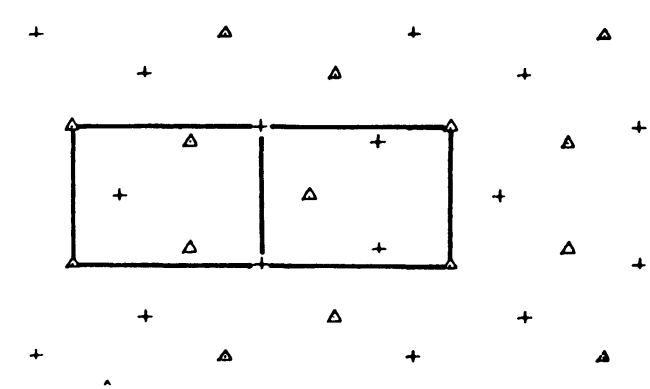

Fig. 3. - [001] symmetrical tilt boundaries in $\mathrm{Cu}(+$ and $\Delta$ represent atoms in different (002) planes : a and b) two structural units may be defined for the favoured boundary $\Sigma=5$ (210), 36 $87 /[001]$ : unit B (a), unit $\mathrm{B}^{\prime}(\mathrm{b}) ; \mathrm{c}$, d and
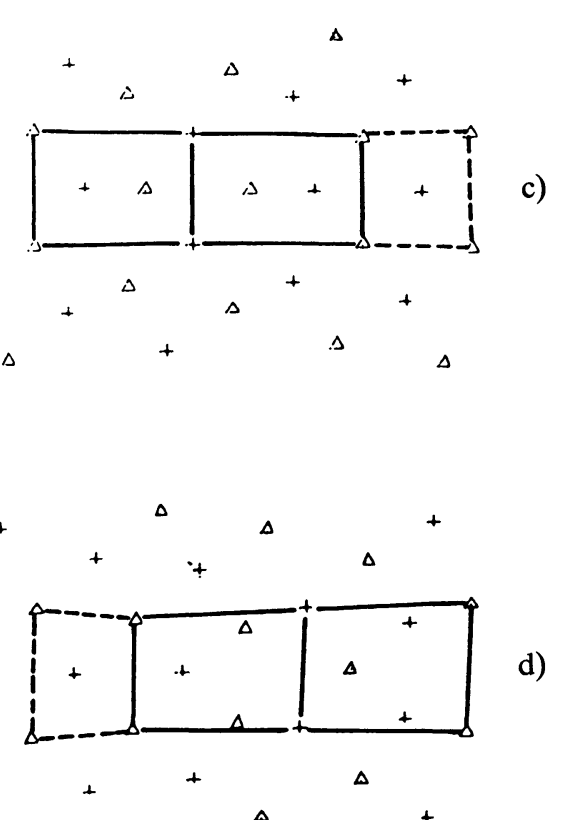

d)

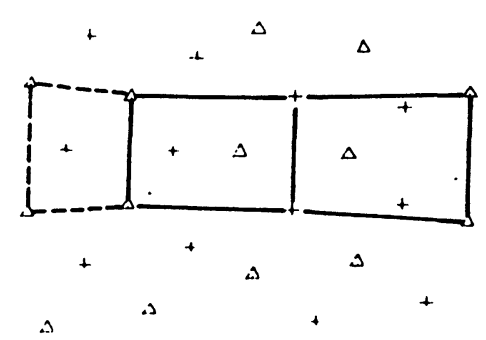

e)

e) different structures of $\Sigma=17$ (530), 28 $07 /[001]$ obtained by combination of the unit of the ideal crystal $\Sigma=1$ (A unit) with the $B$ and $B^{\prime}$ units of the $\Sigma=5$ boundary [4].

proportional to the interplanar spacing $d_{(h k \ell)}$. Paidar $[35,36]$ has lately proposed a classification of symmetrical tilt boundaries $(\langle 110\rangle$ and $\langle 100\rangle$ in the f.c.c. system and $\langle 100\rangle,\langle 111\rangle$ and $\langle 110\rangle$ in the b.c.c. system), based solely on consideration of the reduced interplanar spacings $d / a$ (where $a=$ lattice parameter). An example of the geometrical construction of this classification, tree - generated mathematically, is shown in figure 5. A hierarchy of the different interplanar spacings and its various levels were then defined (tab. II).

This geometrical approach is totally unrelated to the CSL model, but it is striking that the results based on the $d_{(h k \ell)}$ criterion are in good agreement with those of the SU/GBD description. Kwan and Baluffi [33] have recently described many [001] symmetrical tilt boundaries with misorientations ranging from 17 to $73^{\circ}$ as combinations of basic units corresponding to several delimiting boundaries, table I. It should be noted that the first delimiting
Fig. 4. - The first three hierarchical descriptions of the $\Sigma$ 73 (830) [001] symmetrical tilt boundary according to SU/GBD model [33] : a) $\Sigma 1$ (110) minority units plus $\Sigma 1$ (100) majority units; b) $\Sigma 1$ (100) minority units plus $\Sigma 5$ (210) majority units; c) $\Sigma 5$ (210) minority units plus $\Sigma 5$ (310) majority units.

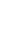




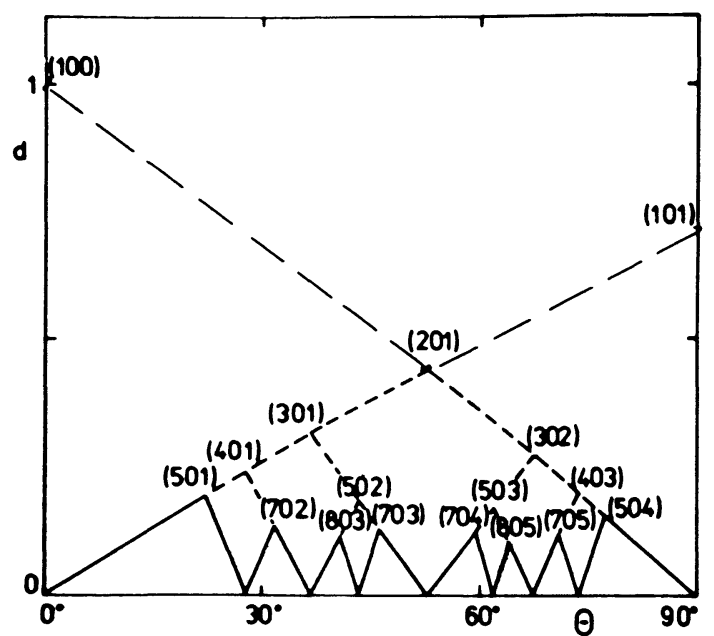

Fig. 5. - Dependance of the interplanar spacings $d$ (in a/2 units) on the misorientation angle $\theta$ for the [010] rotation axis, for the planes in FCC lattice. The classification pyramids are drawn in dashed lines for the planes marked in the figure [36].

Table II. - Classification of symmetrical [010] grain boundary planes in f.c.c. lattice.

\begin{tabular}{|c|c|c|}
\hline Level & Plane & $\Sigma$ \\
\hline 1st & $\begin{array}{l}(100) \\
(101)\end{array}$ & 1 \\
\hline 2nd & $(012)$ & 5 \\
\hline 3rd & $(013)$ & 5 \\
& $(023)$ & 13 \\
\hline 4th & $(014)$ & 17 \\
\hline
\end{tabular}

boundaries chosen appear in the first levels of the Paidar classification [36], table II. The model therefore appears to offer a simple means of selecting possible delimiting boundaries, among which the effective favoured boundaries can only be determined by computer simulations of the intergranular structure.

This construction is only possible because, in the case of symmetrical tilt boundaries, the interplanar spacing data contains the necessary information on the misorientation. What, then, is the situation for a general grain boundary?

In order to study the validity of the $d_{(h k \ell)}$ criterion for asymmetrical boundaries, the planar atomic density can be estimated by considering a mean value of the interplanar spacings [34]:

$$
d_{\text {eff }}=\left[d_{\left(h_{1} k_{1} \ell_{1}\right)}+d_{\left(h_{2} k_{2} \ell_{2}\right)}\right] / 2 .
$$

A new criterion can then be formulated, based on the necessity for a boundary to lie in a plane with a high $d_{\text {eff }}$ value in order to have a strong probability of speciality. As in the preceding models, in which limiting values were considered for $\Sigma, \Delta \theta$ or for the periodicity of the structural units, in the present case an arbitrary minimum value must be defined for the reduced interplanar spacing $(d / a)_{c}$. Above this value, it can be reasonably assumed that the intergranular region is not greatly distorted and that the boundary may present special properties, although no correlation has yet been established experimentally.

In practice, the further question arises as to whether or not the coincidence defined by $\Sigma$ is related to the orientation of the boundary plane, or more precisely, as to whether a boundary classified as « general » due to its high $\Sigma$ or $\Delta \theta$ values can have a high atomic planar density. Although there is theoretically no necessity for the position of the interface to be related to the geometric relation between the grains, it is not impossible that grain growth mechanisms impose boundary orientations which are not totally independent of the prevailing distribution of misorientations.

2.4 ConClusion. - Whatever the approach to speciality, there remains the common underlying question of whether certain grain boundaries can show particular behaviour. Indeed, it is usually assumed, either explicitly or implicitly, that the properties of a boundary depend on its structure, based solely on a knowledge of its geometry. This hypothesis remains true in the case of the structural unit model, even if atomic relaxations are permitted. The inability of these models to provide a completely satisfactory answer to this question arises because they neglect the essential fact that the properties of a boundary, like those of a crystal, probably depend more on defects than on the «ideal " structure. Among these defects, the interstitial and substitutional atoms segregated to the grain boundary (impurities or deliberate alloying additions) play a predominant role. Before undertaking any investigation of grain boundary properties, it is therefore essential to thoroughly understand the relationship between segregation and intergranular structure.

A given behaviour may be related to the intrinsic structure of a boundary, or may depend on the suitability for segregation induced by a particular intergranular geometry. Grain boundary properties are thus determined by an indivisible combination of intergranular geometry and chemistry. Moreover, the presence of substitutional or interstitial segregation can modify the distribution of boundary structures within a polycrystalline material.

For this reason, the only valid approach to the problem of speciality is to distinguish between 
boundaries in terms of their effective properties, taking into account both their crystallography and their composition. At first, such distinctions can only be specific, and will vary with the property considered and from one material to another.

The aim of the following section is to summarize the results obtained using this approach and to try to establish the relationship between certain intergranular properties, including the segregation response, and the geometrical characteristics of the grain boundaries. Grain boundary energy, which has been treated in a recent review article [37], will not be considered.

\section{Special properties and grain boundary geometry.}

The relationship between the properties and the crystallographic characteristics of grain boundaries has been studied mainly on bicrystals. Such investigations are useful for interpreting specific mechanisms ; furthermore, they single out certain symmetrical tilt boundaries, with well-defined planes related to misorientations. They do not, however, give any information on the possible overriding influence of a particular geometrical parameter on a given property. More especially, because of the limited number of boundaries analyzed and their «ideal » isolated structure, the results obtained bear little relevance to the behaviour of random boundaries in real polycrystalline materials. For this reason, the information gained from bicrystal studies will be recalled only briefly, before treating in more detail the results of recent work on polycrystals. In this context, properties which have been found to depend chiefly on misorientation will be distinguished from those for which the orientation of the boundary plane appears to be important. With this relatively arbitrary distinction, the properties can be considered in two groups :

- mechanical properties such as low temperature intergranular fracture, or cavitation and grain boundary sliding at high temperatures ;

- properties more closely related to the characteristics of the boundary core, such as the segregation behaviour (which strongly affects the intergranular corrosion resistance) and grain boundary diffusion.

\subsection{SPECIAL PROPERTIES AND MISORIENTATION.}

3.1.1 Intergranular fracture. - Experiments on gallium-induced intergranular fracture have been carried out on zinc and aluminium bicrystals [38]. The stress required for failure was found to be significantly higher for boundaries with low misorientations or for those close to positions with relatively low coincidence values $(\Sigma=3$ and 11 for $\mathrm{Al}$, and $\Sigma=9$ for $\mathrm{Zn}$ ). The same method applied to polycrystalline copper, in which grain boundary orientations were determined using the electron channeling pattern (ECP) technique, showed that «general» boundaries (in this case $\Sigma>25$ ) act as preferential paths for fracture propagation, the latter becoming transgranular when a special boundary is encountered [39].

Two fracture processes can then be envisaged, depending on the distribution of grain boundaries in the polycrystal, figure 6 [7]. Several years after its

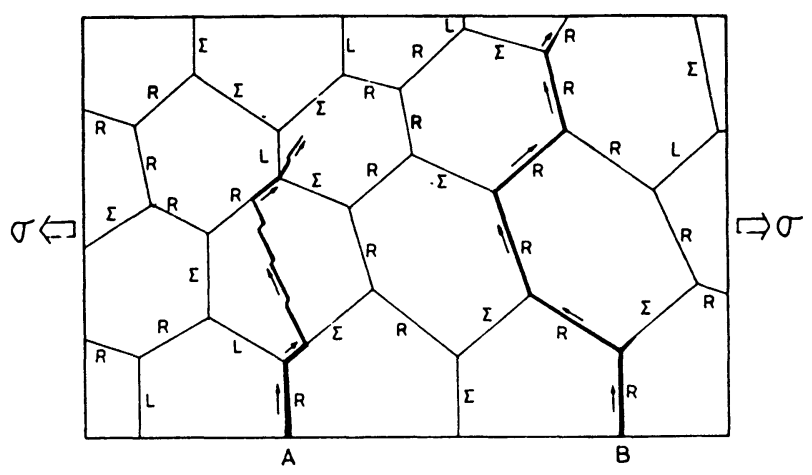

Fig. 6. - Schematic representation of grain boundary structure dependant fracture processes in polycrystals: Path A: Combined process of intergranular and transgranular fracture ; path B : Typical intergranular fracture. The fracture follows the path of "general » boundaries here called $\mathrm{R}$ (for Random) [7].
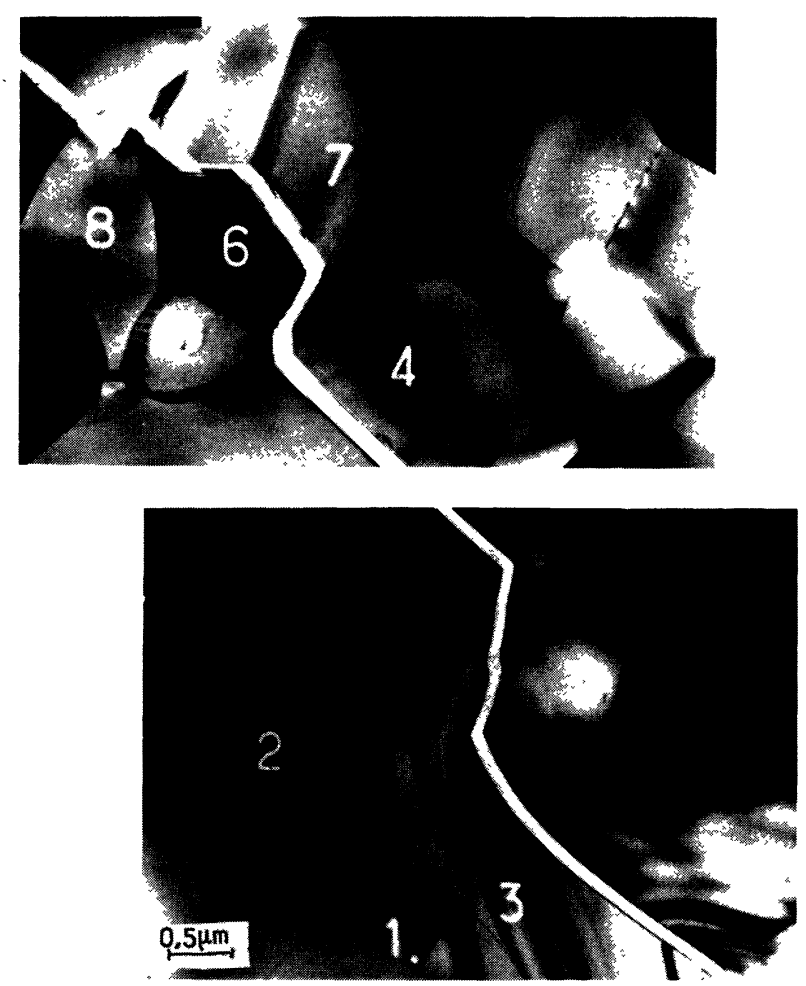

Fig. 7. - Electron Micrograph showing a crack in a deformed sample of alumina. The crack is following general boundaries; it becomes intragranular when meeting a special boundary $2 / 3(\Sigma 17$ b) [40]. 


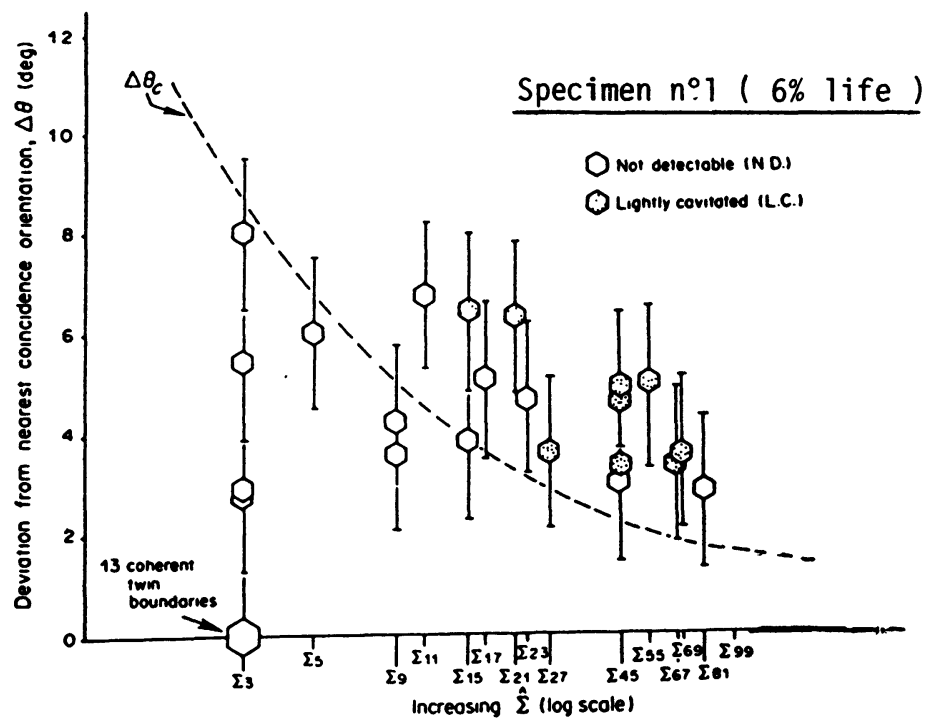

(a)

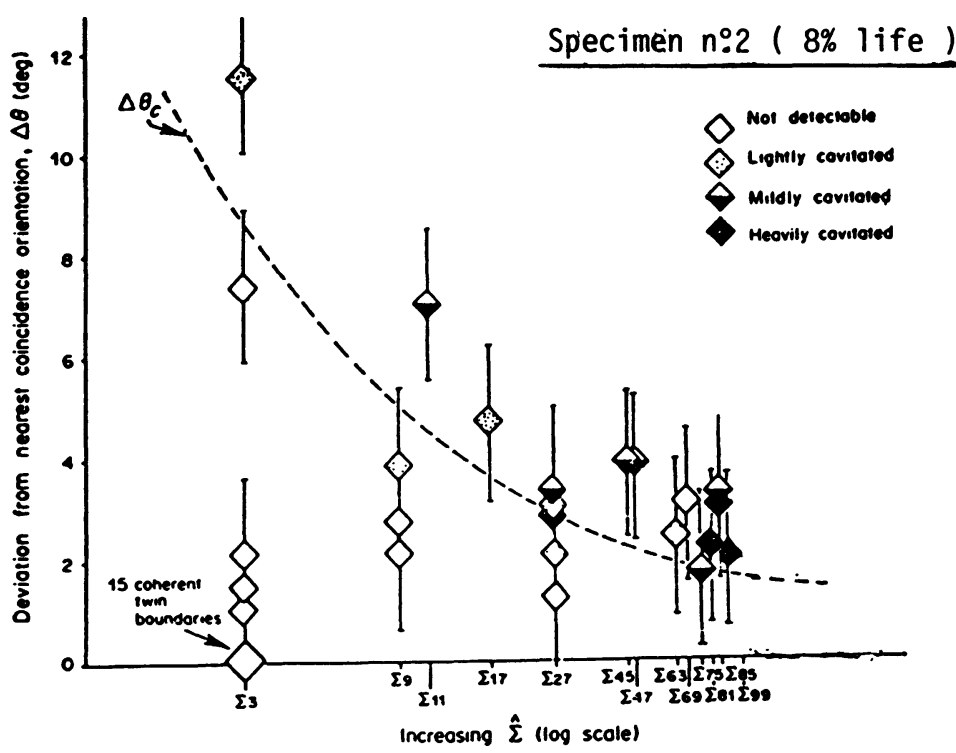

(b)

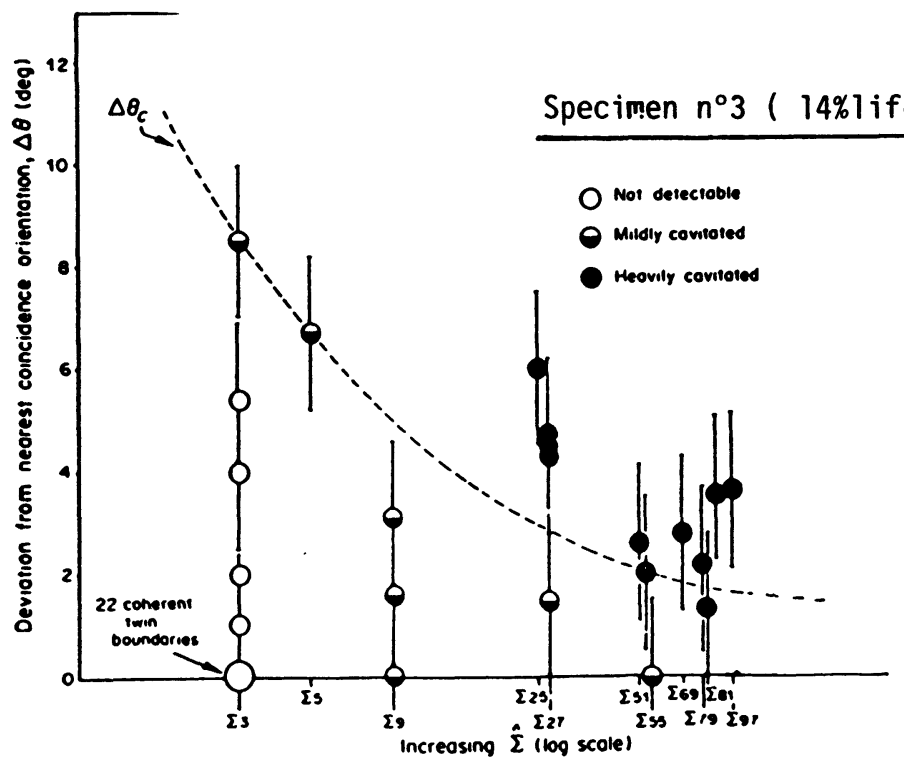

(c)

Fig. 8. - Effect of $(\Sigma, \Delta \theta)$ on cavitation in Nickel specimens interrupted at : a) $6 \%$ life fraction ; b) $8 \%$ life fraction ; c) $14 \%$ life fraction. The critical deviation of Brandon $\Delta \theta_{c}$ versus the $\Sigma$ value is also reported (dashed line) [41]. 
proposal by Watanabe, this representation was justified by observations on the spontaneous low temperature fracture of polycrystalline alumina, figure 7 [40].

3.1.2 Intergranular cavitation. - The most systematic study of the relationship between fatigue-induced intergranular cavitation and grain boundary structure was carried out by Lim and Raj on polycrystalline specimens of pure nickel [41]. The misorientation parameters were determined by $\mathrm{X}$ ray diffraction, and boundaries were considered to be «special» when $\Sigma<99$ and $\Delta \theta_{\exp }<\Delta \theta_{\mathrm{c}}, \Delta \theta_{\mathrm{c}}$ being defined according to Brandon's criterion [24]. The results obtained for fractional lifetimes of 6,8 and $14 \%$ are illustrated in figure 8 . The application of this criterion enables the cavitation-sensitive boundaries to be roughly differentiated whatever the duration of fatigue testing, cavities appearing first in boundaries with high $\Sigma$ or $\Delta \theta$ values. When cycling is continued to failure, only coherent twin boundaries $(\Sigma=3,\{111\})$ are cavity-free. Some incoherent segments of such boundaries $(\Sigma=3)$ already show cavitation for fractional lifetimes of $14 \%$. The two latter observations indicate that the orientation of the boundary plane is a second factor which can affect cavitation.

One of the mechanisms invoked to explain cavity nucleation is the formation of extrinsic dislocation pile-ups at grain boundary irregularities (see Sect. 3.2.3).

3.1.3 Grain boundary sliding. - Grain boundary sliding is undoubtedly the deformation phenomenon which has been most widely studied in relation to intergranular structure. About 15 years ago, the influence of the relative misorientation of the two grains was revealed almost simultaneously during studies of intergranular creep on bicrystals of aluminium and copper [42] and stainless steel [43]. The results obtained on these different materials were in good agreement and showed much higher creep rates (by 2 to 3 orders of magnitude) in boundaries very close to coincidence positions (Fig. 9), the difference decreasing at higher temperatures (Fig. 10). Plots of the sliding displacement $S$ as a function of misorientation also presented pronounced minima for certain coincidence positions.

This marked influence of misorientation on intergranular creep seems to indicate that the mechanism involved is not simply a viscous process. By analogy with creep mechanisms within the grains, the authors invoke the role of extrinsic dislocations, intergranular structural defects similar to lattice dislocations. Computer simulations of the creation and migration of a dislocation in a grain boundary lead to formation and displacement energies lower than for a lattice dislocation, and therefore support the hypothesis that one of the fundamental mechanisms of grain

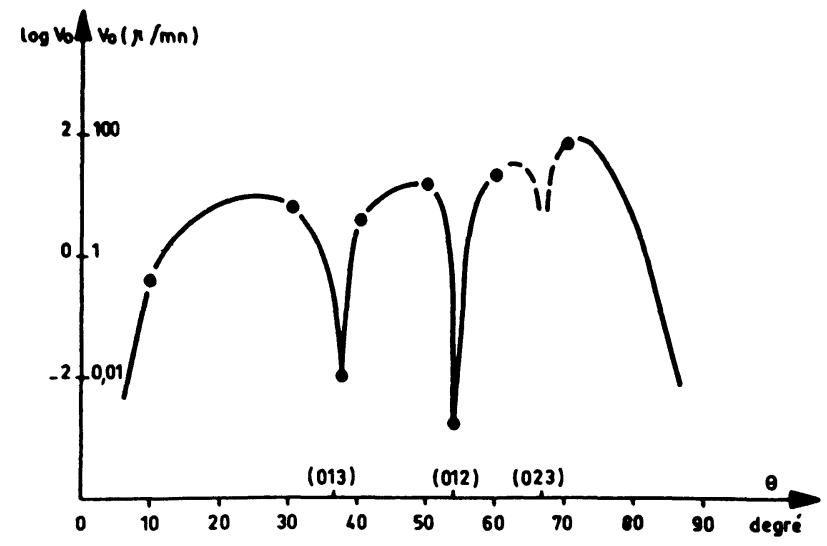

Fig. 9. - Misorientation dependence of the initial rate of sliding for copper bicrystals $(\langle 100\rangle$ tilt grain boundaries - Creep stress parallel to the rotation axis $=1 \mathrm{MPa}-$ $T^{\circ}=600^{\circ} \mathrm{C}$ ) [42].

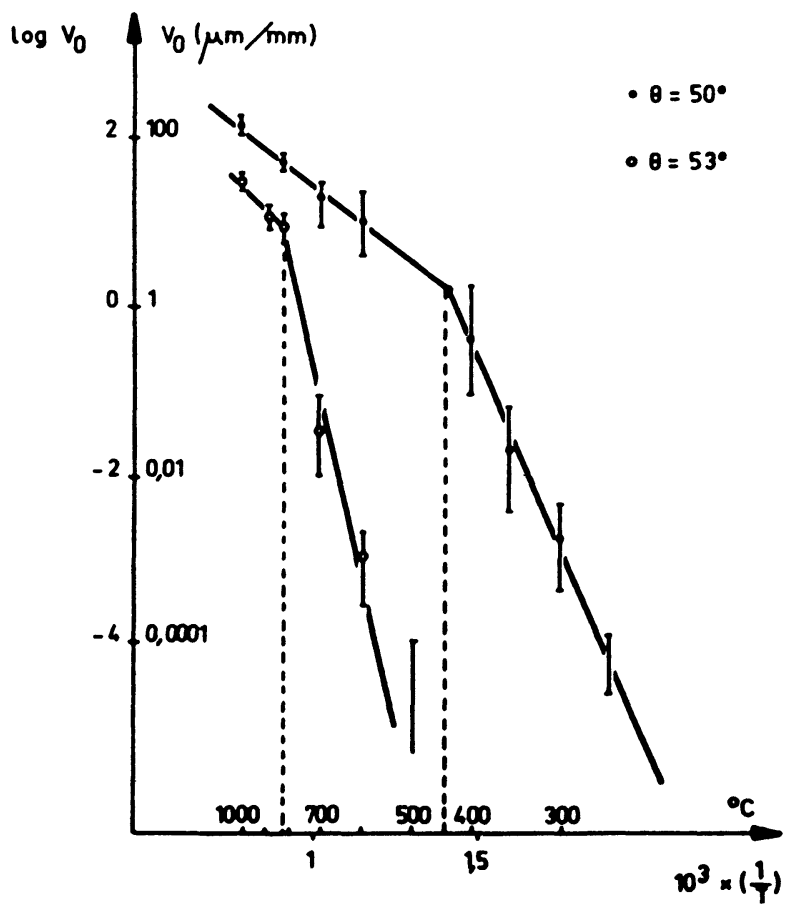

Fig. 10. - Temperature dependence of the initial rate of sliging for copper bicrystals. The $53^{\circ}$ misorientation is very close a CSL boundary with $\Sigma=5$. The $50^{\circ}$ misorientation may be considered as a " general » boundary (see Fig. 9) ( $T$ and $T^{\circ}$ similar to those of Fig. 9).

boundary sliding is the movement of extrinsic dislocations in the boundary plane [44].

The preceding results obtained on bicrystals, together with the processes which they suggest, were confirmed by studies on the creep behaviour of grain boundaries in coarse-grained polycrystals (Fig. 11) $[7,45]$. Boundaries which were little prone to sliding 


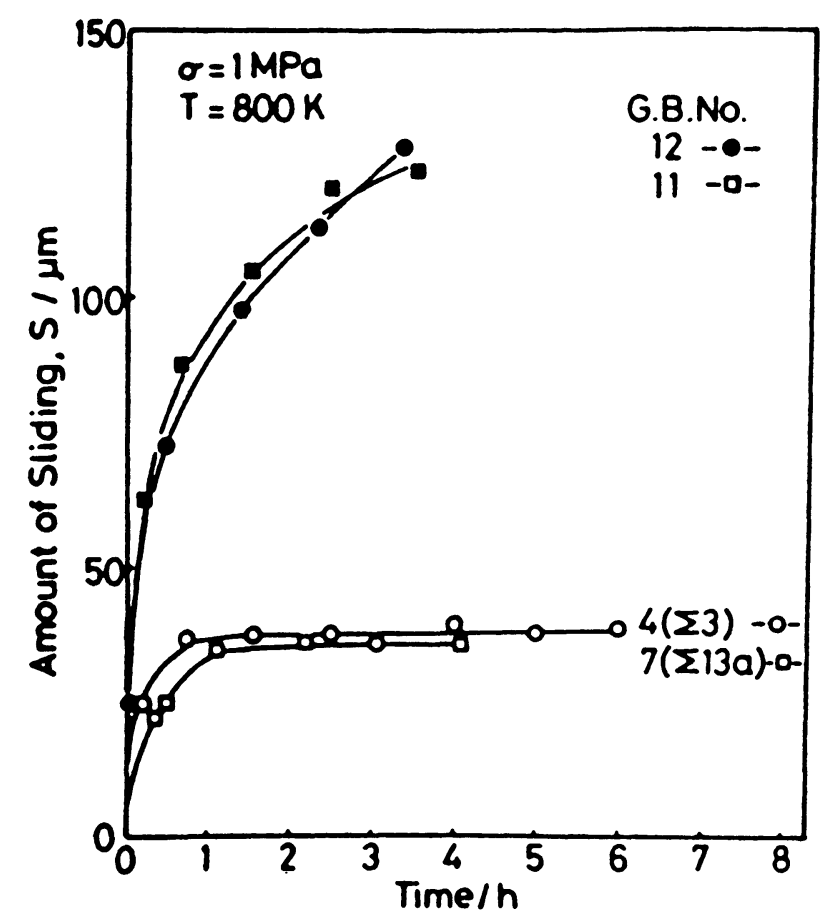

Fig. 11. - Sliding curves for general boundaries $\left(\mathrm{n}^{\circ} 11\right.$ and $n^{\circ} 12$ ) and for coincidence boundaries ( $n^{\circ} 4$ and $\left.n^{\circ} 7\right)$ in aluminium polycrystals [7].

were found to have special misorientations close to coincidence positions, and respected Brandon's criterion [24]. Contrary to «general » boundaries, they presented extrinsic dislocations after deformation (Fig. 12). These observations support the model for intergranular creep proposed by Horton and co-

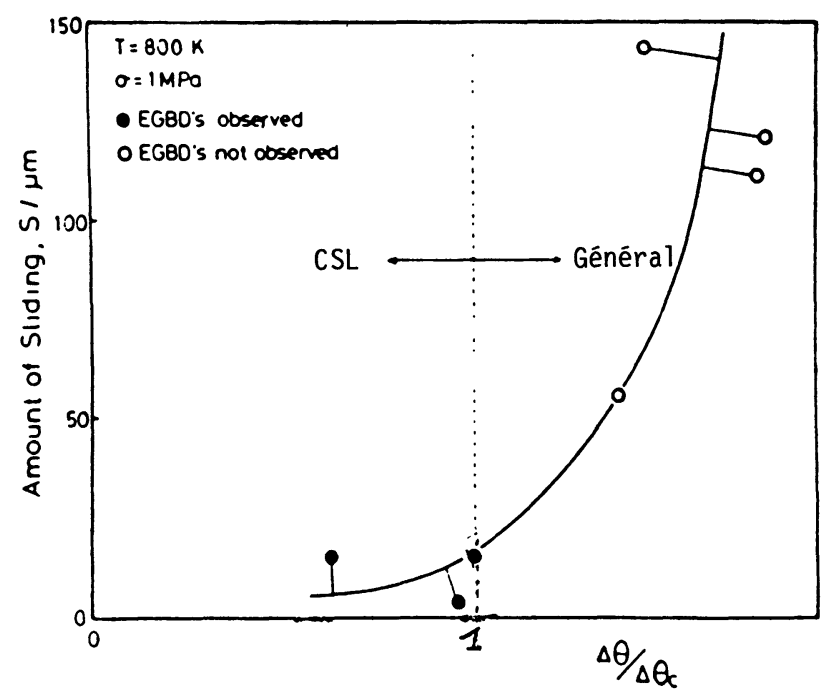

Fig. 12. - Relationship between the amount of sliding $S$ and the degree of angular deviation from the nearest coincidence orientation given by $\Delta \theta_{\exp } / \Delta \theta_{\mathrm{c}}$ in which $\Delta \theta_{\exp }=\theta_{\exp }-\theta_{\mathrm{CSL}}$ and $\Delta \theta_{\mathrm{c}}=$ critical deviation of Brandon. After sliding, extrinsic dislocations are not observed in general boundaries. workers [46], in which lattice dislocations are absorbed into the boundary, and the extrinsic dislocations resulting from this interaction, which can vary from one boundary to another, then determine the sliding behaviour. In order to gain a better understanding of intergranular creep, it is therefore essential to study the relationship between the behaviour of extrinsic dislocations and the geometry of the boundaries in which they are formed (see Sect. 3.2.3).

\subsection{SPECIAL PROPERTIES AND BOUNDARY PLANE ORIENTATION.}

3.2.1 Intergranular segregation. - Except for a change in structure observed in a twist boundary in gold, and attributed to the presence of iron [47], there has apparently been no systematic experimental work on the relationship between segregation and boundary structure in bicrystals. On the contrary, calculations of segregation energies have enabled the prediction of favourable sites for the insertion of interstitial or substitutional atoms into the structural unit of a boundary, depending on its orientation [48-50]. The configurations obtained can account for the embrittling or strengthening effects produced [49-51].

Recent studies on polycrystals emphasize the dominant role of boundary orientation on segregation behaviour. Thus, Auger spectroscopy measurements showed that the quantity of phosphorus segregated to grain boundaries in iron depends on the crystallographic indices of the boundary plane. Large concentrations were found if the interface has high indices in both crystals, whereas segregation was only slight for low indices. Different amounts of phosphorus were often observed on the two fracture surfaces of a same boundary, depending on the indices of the corresponding surfaces [52]. Variations observed in the sensitivity of a steel to intergranular attack by a reagent which selectively etches phosphorus-rich regions lead to a similar conclusion [53].

A detailed study of sulphur segregation in the grain boundaries of nickel yielded a more precise relationship, based on the planar atomic density criterion (cf. Sect. 2.3). The reduced interplanar spacing must apparently exceed a minimum value $(d / a)_{\mathrm{m}}$ for the boundary to be relatively little prone to segregation. The latter is revealed by a technique involving local preferential etching of sulphur-rich boundaries in thin foils (Fig. 13) [54, 55].

If the parameter $(d / a)$ is plotted as a function of $\Sigma$ for symmetrical tilt boundaries in the f.c.c. structure, such that the density of coincident sites is equal to the density of atomic sites in the interface $(\sigma=1)$ (Fig. 14) it is interesting to note that a critical value $(d / a)_{\mathrm{c}} \cong 0.150$ deduced from the observations on 


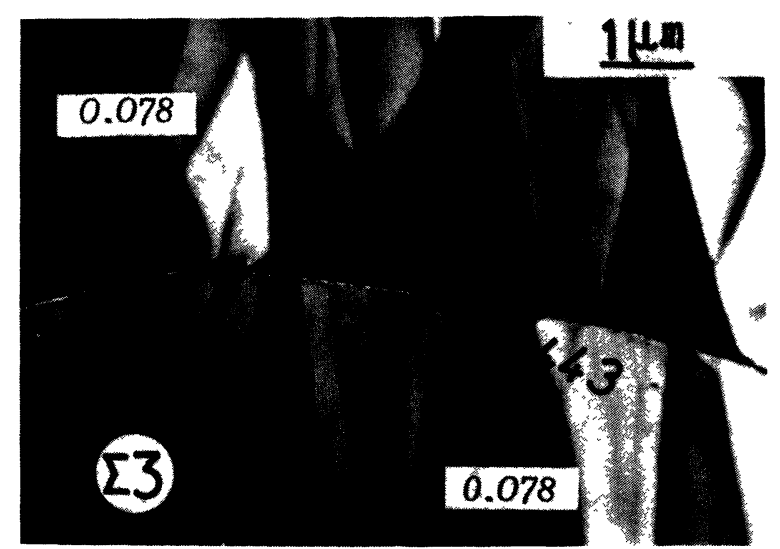

a)

Fig. 13. - Observation by TEM of the microetchings of a special $\Sigma=3$ grain boundary with a curved plane (d/a values reported in italic characters) : a) microetched part ;

nickel favours the first levels of the Paidar classification [35, 36] (cf. Sect. 2.3).

Finally, the reciprocity of the relationship between the planar atomic density and the concentration of segregated elements is supported by an experiment

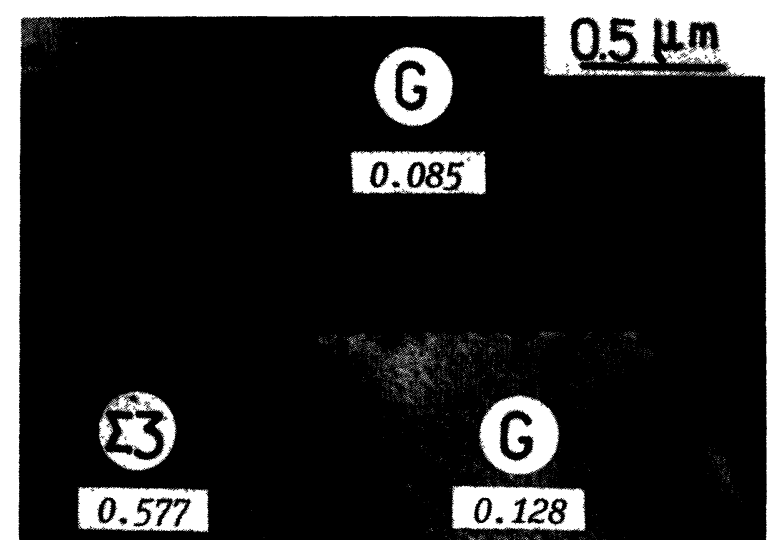

b)

b) non microetched part (prolongation of the region a). Note the two general boundaries are microetched [55].

in which the desulphurization of a nickel thin foil was observed to change the orientation of a boundary close to the $\Sigma=3$ coincidence position, in such a way as to increase its atomic density. In turn, a further sulfurization leads to a decrease of the

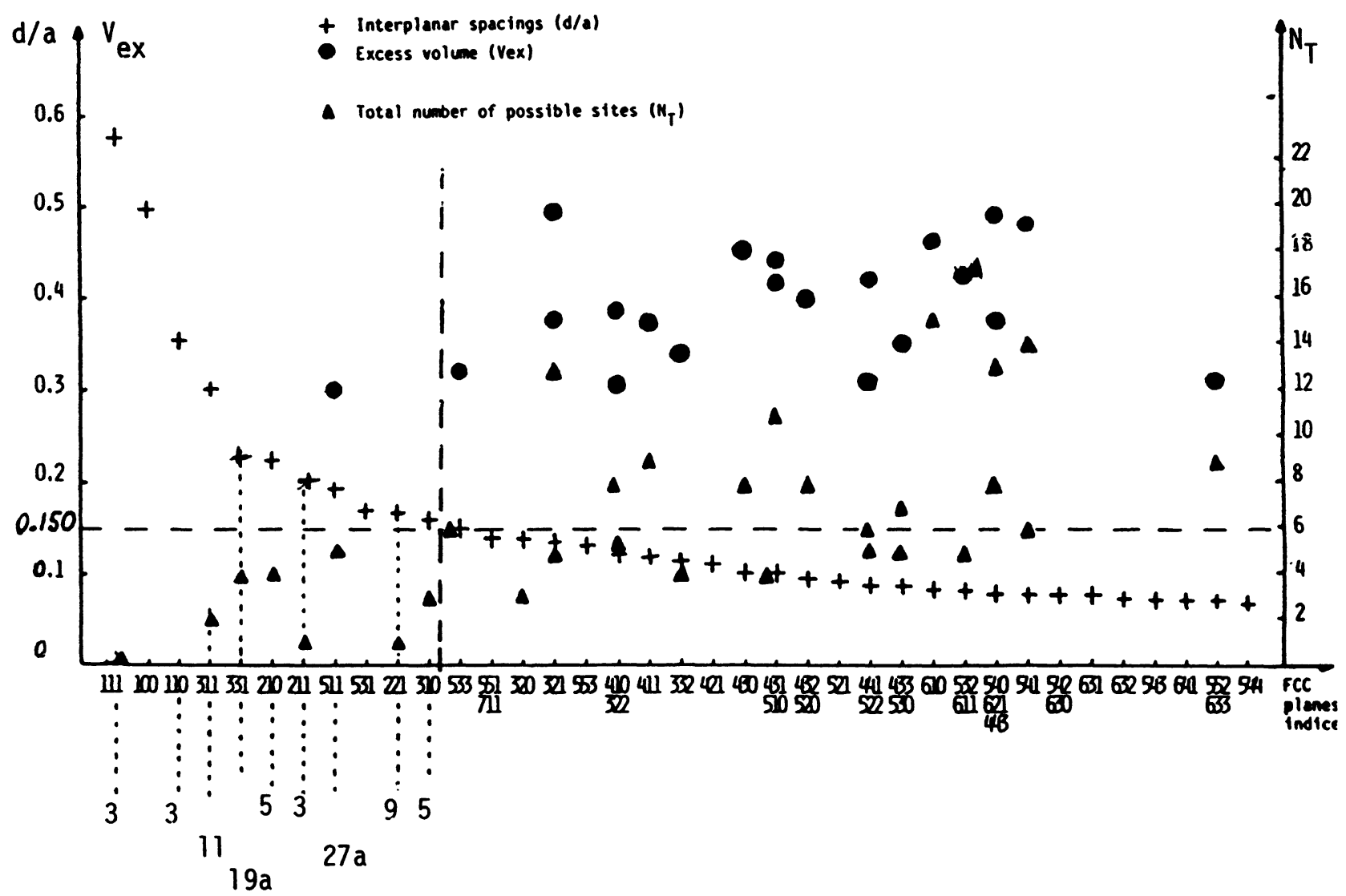

Fig. 14. - Interplanar spacings, excess volumes and total numbers of interstitial and substitutional sites as functions of $\Sigma$ for symmetrical $\langle 100\rangle,\langle 110\rangle$ and $\langle 111\rangle$ tilt boundaries in FCC structure $(\sigma=1)$. A transition zone may be experimentally located around the value $d / a=0.150$ [55]. The segregation becomes very low for $d / a$ superior to this value corresponding to the grain boundary planes of the 2nd and 3rd level in the Paidar classification [36]. 
atomic density [56]. A knowledge of the intergranular segregation is essential, since it modifies the thermodynamic characteristics of a boundary by introducing new variables, the concentration and the chemical potential of each element, while at the same time exerting a strong influence on the majority of the physical phenomena controlled by intergranular diffusion. It is highly probable that the differences related to geometry are greatly attenuated in « impure " boundaries, with only coherent twin boundaries $(\Sigma=3)$, which are impermeable to segregation, conserving their special behaviour.

Finally, it should be noted that, for polycrystalline materials produced in a similar manner, variations in purity can affect the distribution of grain boundaries with different geometrical characteristics (cf. Sect. 4).

\subsubsection{Intergranular diffusion: direct studies on bic-} rystals. - The idea that grain boundary diffusion is controlled by the jump efficiency of point defects in the boundary was investigated about fifteen years ago [57], and the intergranular diffusion coefficient appears to be the macroscopic parameter which is the most closely related to the boundary core structure. However, because of the diversity of atomic configurations, even in the case of perfectly controlled bicrystals, and in the simplest case of selfdiffusion, it is extremely difficult to analyze the relationship between diffusion and structure.

Nevertheless, the jump mechanism has been supported by both experimental observations and by theoretical calculations of intergranular diffusion coefficients, based on static and dynamic structural models. From the experimental standpoint, a marked anisotropy is observed in the diffusion coefficients $D_{\mathrm{b}}$, depending on whether they are measured parallel or perpendicular to the tilt axis in boundaries of low misorientation $[58,59]$. Remarkably low values of $D_{\mathrm{b}} \cdot \delta$ (where $\delta$ is the boundary width) have been found for misorientations corresponding to $\Sigma=3,[111]$ and $\Sigma=11$, [113] in various f.c.c. metals [59]. The correlation between measured and calculated $D_{\mathrm{b}}$ values is quite good (Fig. 15) [58-60]. For a given misorientation, the diffusion coefficient varies with the deviation from the symmetrical position [61].

More recently, the determination of the binding energies associated with different sites, which control the jump frequency, has enabled intergranular diffusion paths to be predicted [62-63]. The jump efficiency appears to be independent of the type of boundary [63], but for a given misorientation, differs according to the orientation of the boundary plane [60], in agreement with previous experiments [61].

3.2.3 Intergranular diffusion : indirect measurements on polycrystals. - The indirect measurement of the diffusion coefficient in a polycrystal grain boundary

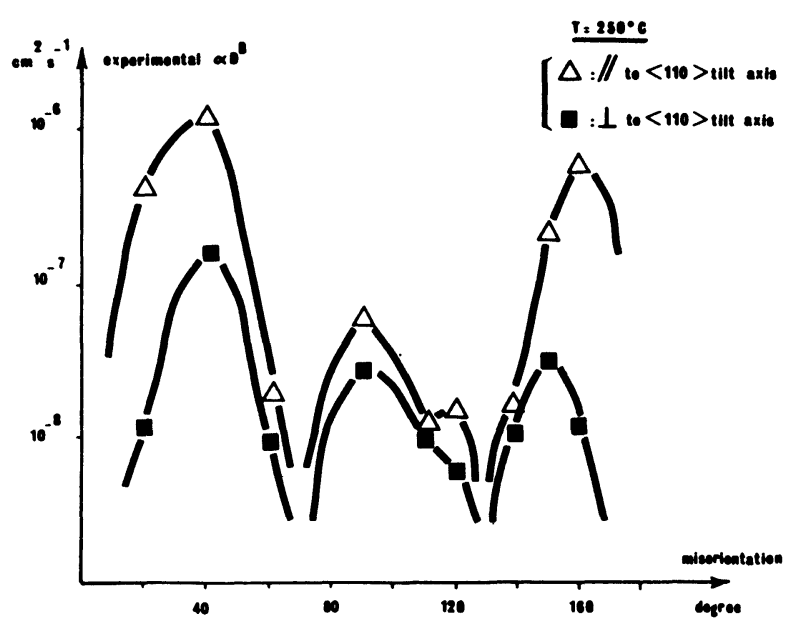

a)

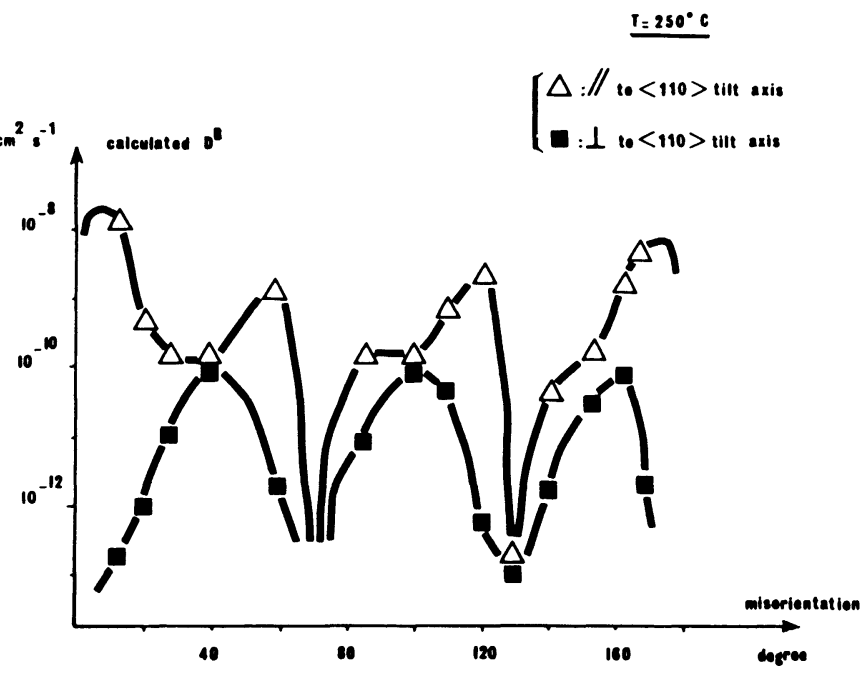

b)

Fig. 15. - Experimental apparent intergranular diffusion coefficients of zinc at $250^{\circ} \mathrm{C}$ (a) and calculated intergranular self-diffusion coefficients at $250^{\circ} \mathrm{C}$ (b) in symmetrical tilt boundaries around $\langle 110\rangle$ in aluminium : influence of the misorientation and of the direction of diffusion [60].

is based on the phenomenon of thermally activated accommodation of lattice dislocations incorporated into the boundary. The processes by which a grain boundary containing extrinsic dislocations returns to an equilibrium state have been predicted theoretically [64] and explained on the basis of experimental observations $[65,66]$. The fundamental mechanism is the dissociation of the matrix dislocation via the reaction :

$$
b_{\mathrm{m}} \rightarrow n b_{\mathrm{DSC}}
$$

followed by separation of the products. In most polycrystal boundaries, $n$ is large (i.e. $b_{\mathrm{DSC}}$ is small), so that the dissociation can alternatively be considered as a broadening of the dislocation core (Fig. 16) [66-67]. 

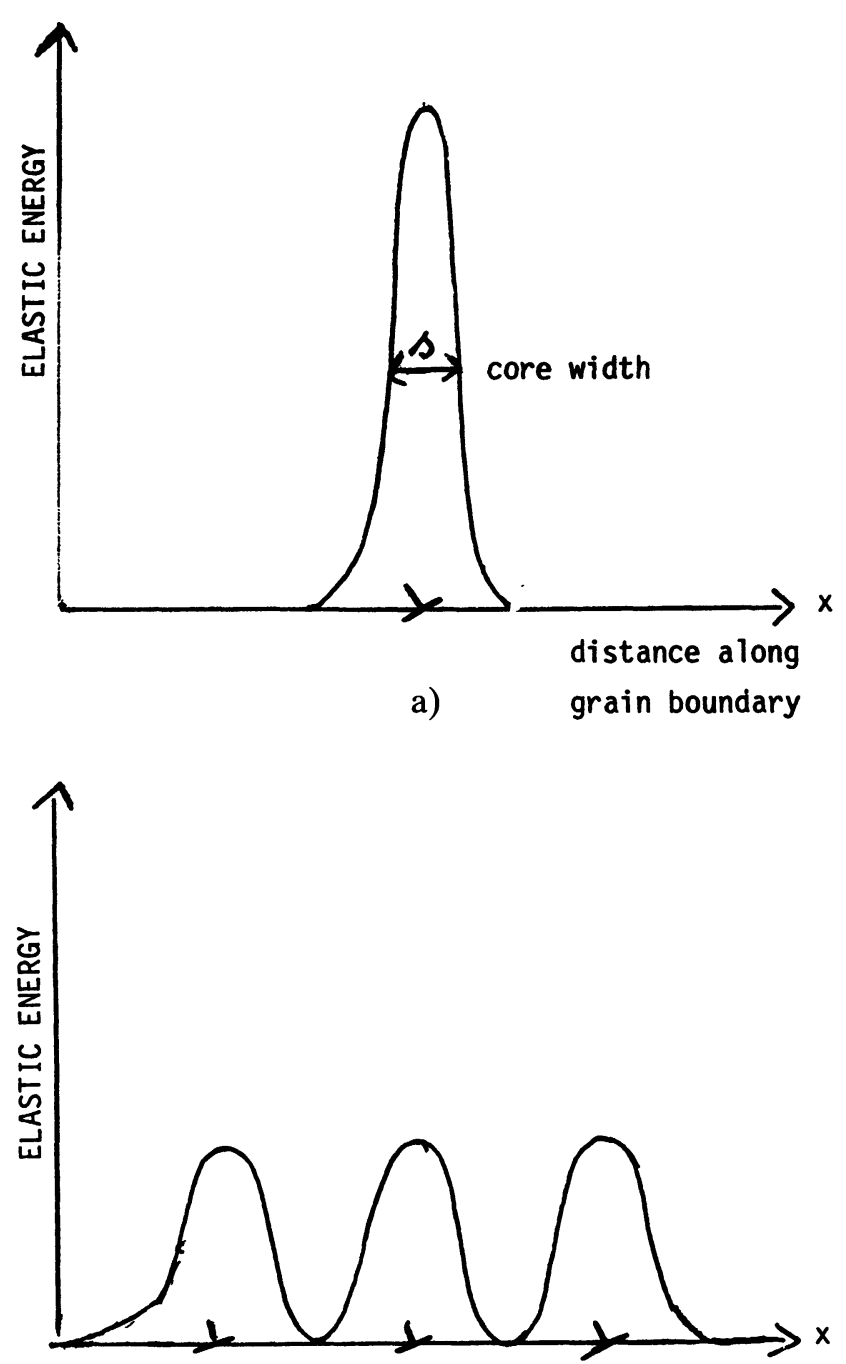

b)

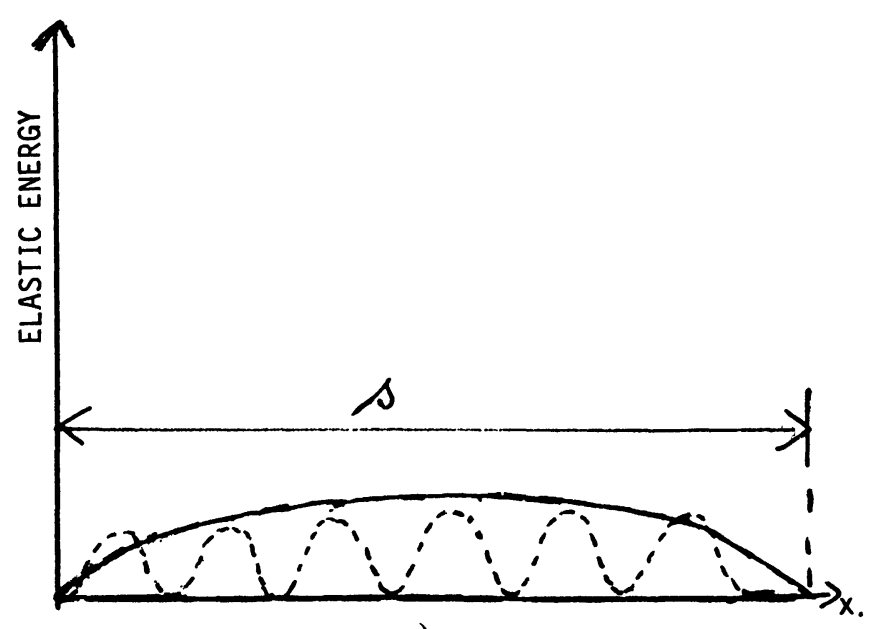

c)

Fig. 16. - Schematic representation of the accommodation of extrinsic dislocations : a) initial state : extrinsic dislocation localized in the grain boundary with $s=$ core width and $b=b_{\mathrm{m}} ; \mathrm{b}$ ) discontinuous process of accommodation : dissociation $\left.b_{\mathrm{m}}=n b_{\mathrm{DSC}} ; \mathrm{c}\right)$ continuous process of accommodation: broadening of the dislocation core $s$. If $n \rightarrow \infty$ i.e. $b \rightarrow 0$, the processes $\mathrm{b}$ and $\mathrm{c}$ are equivalent.
Whatever the interpretation, the kinetics are the same. When the separation $s$ between dislocations (or the core width) approaches two extinction distances $\xi_{j}$, the TEM image contrast disappears [68]. In a given grain boundary, only a few rare dislocations, probably with particularly oriented Burgers vectors, disappear more rapidly than the others (Fig. 17) [69]. However, because the Burgers vectors of extrinsic dislocations do not generally lie in the plane of the boundary, their displacement in the latter requires climb, and this explains their statistically identical behaviour. For each boundary, there will therefore exist a pair of characteristic values $T_{\mathrm{D}}$ and $t_{\mathrm{D}}$, corresponding respectively to the temperature and time for which dislocation contrast disappears in the TEM, and related to intergranular diffusivity as follows :

$$
t_{\mathrm{D}}=A T_{\mathrm{D}} / D_{\mathrm{b}} \delta \quad Q_{\mathrm{b}}=R T_{\mathrm{D}} \log K t_{\mathrm{D}} / T_{\mathrm{D}}
$$

where $A$ and $K$ are constants depending on $s$ and $b_{\mathrm{DSC}}$ and $Q_{\mathrm{b}}$ is the activation energy for grain boundary diffusion.

By assuming that the intergranular dislocation has a Burgers vector $b$ perpendicular to the boundary plane, the values of $D_{\mathrm{b}} \delta$ and $Q_{\mathrm{b}}$ can be calculatęd from the experimental measurements of $T_{\mathrm{D}}$ and $t_{\mathrm{D}}$, which are characteristic of the geometry and purety of the boundary.

This method has been extensively employed by Grabski and co-workers [70], and has enabled them to establish the continuity of the distribution of grain boundary diffusivities in polycrystals (Fig. 18). Furthermore, the mean diffusivity obtained from measurements of $T_{\mathrm{D}}$ and $t_{\mathrm{D}}$, for several boundaries, was found to be compatible with the " macroscopic » value deduced from classical radiotracer experiments [71].

However, it should be noted that, in terms of misorientation, this distribution takes into account only " general » boundaries. In effect, the disappearance of the contrast of dissociated dislocations requires not only their mobility, but also sufficiently small Burgers vectors for them not to be visible individually. Moreover, the above authors point out that certain boundaries conserve their dislocations after prolonged annealing at a relatively high temperature.

In special CSL or CAD boundaries (cf. Sect. 2.1), the Burgers vectors of the dissociated dislocations are no longer negligible and their accommodation can lead to two possible configurations :

- either the accommodation is total, the dislocations forming a regular network and taking on an intrinsic character, in which case there is a change in the boundary misorientation $[64,66]$;

- or the accommodation remains only partial, the extrinsic dislocations taking up a stable pseudoperiodic arrangement in the boundary. 


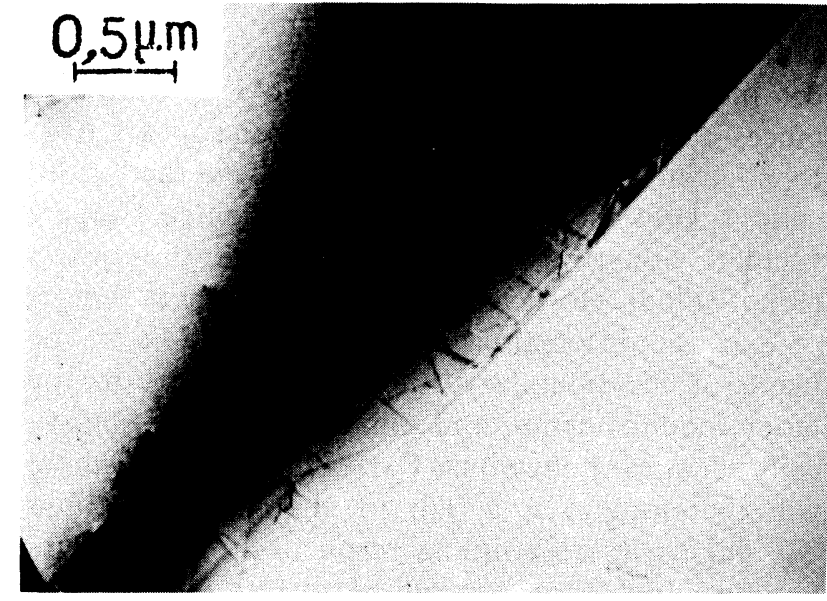

a $\left(20^{\circ} \mathrm{C}\right)$

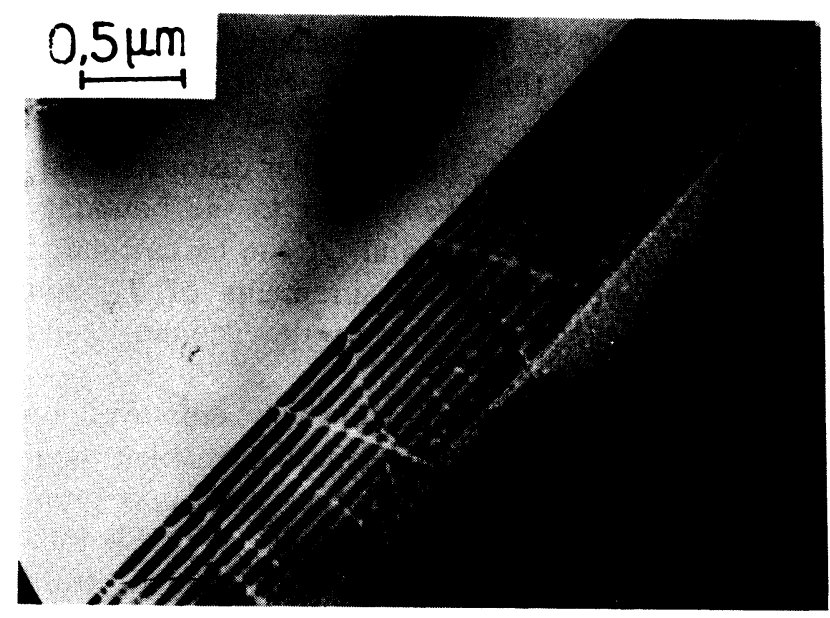

c $\left(420^{\circ} \mathrm{C}\right)$

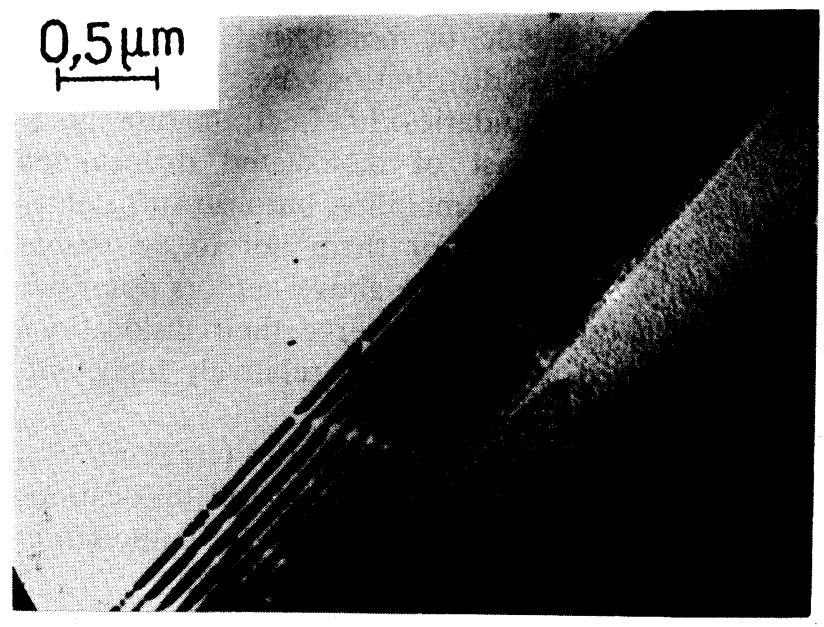

e $\left(470^{\circ} \mathrm{C}\right)$

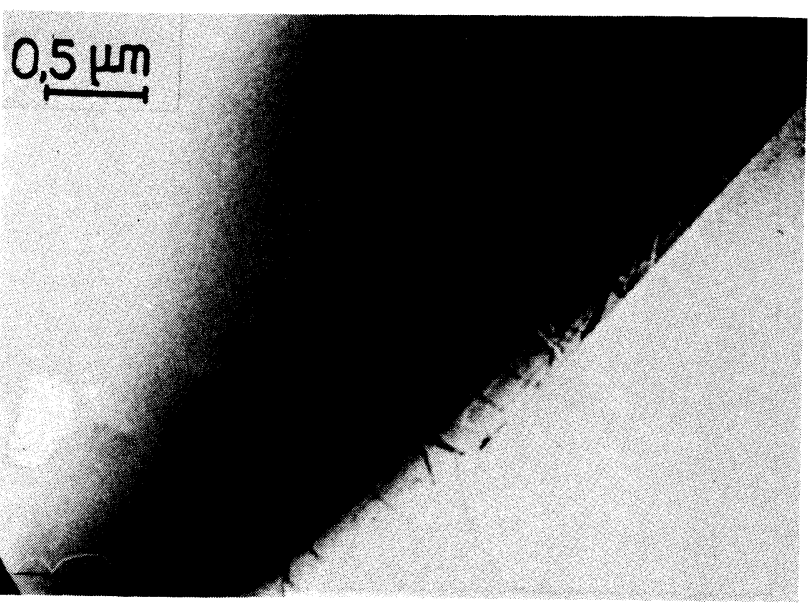

b $\left(380^{\circ} \mathrm{C}\right)$
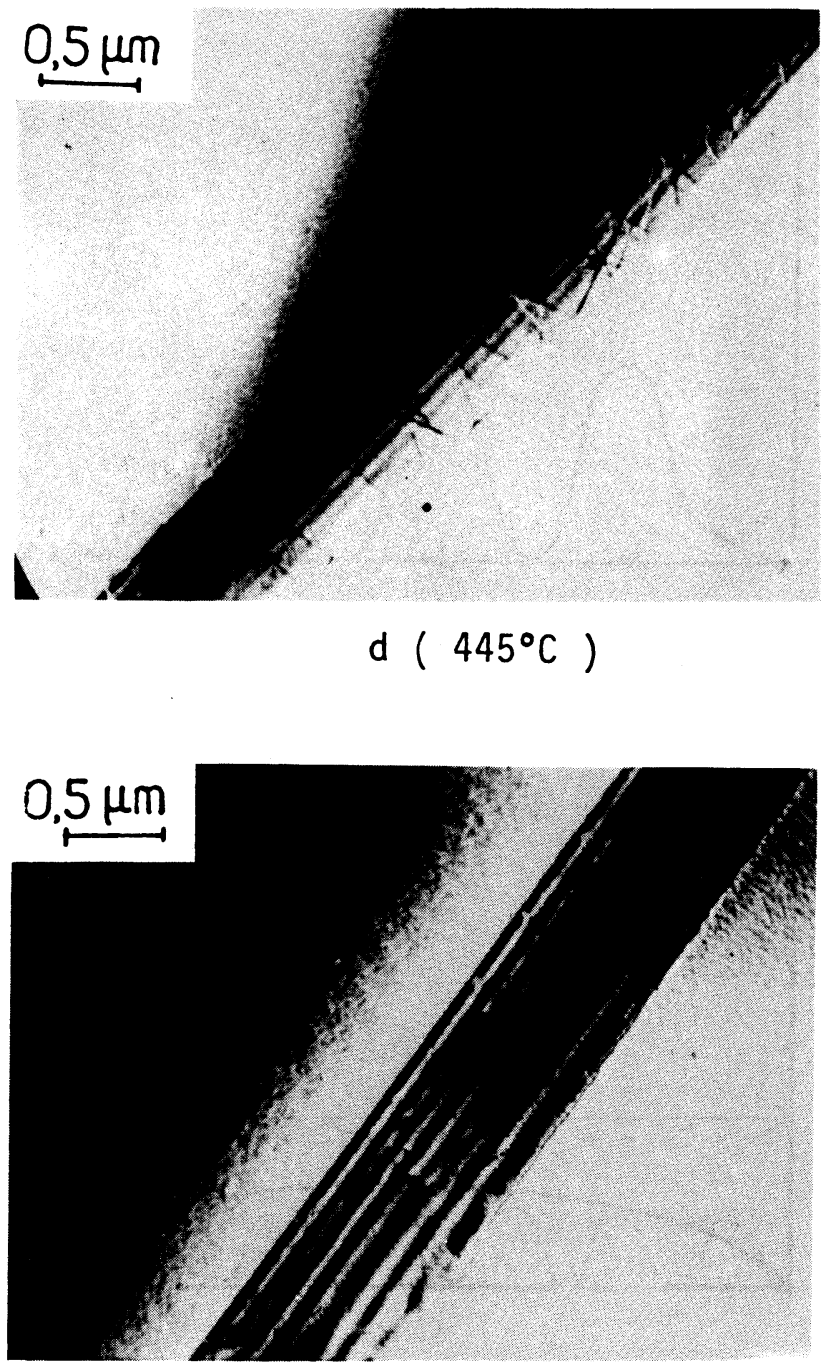

$f\left(510^{\circ} \mathrm{C}\right)$

Fig. 17. - Evolution of the contrast of extrinsic dislocations with temperature observed by in-situ experiments in the High Voltage Electron Microscope (HEVM) a) Room temperature ; b) disappearance of a few rare dislocations (arrowed) ; c, d, e) broadening of the dislocation images between 420 and $470{ }^{\circ} \mathrm{C}$; f) total disappearance of extrinsic dislocations. 


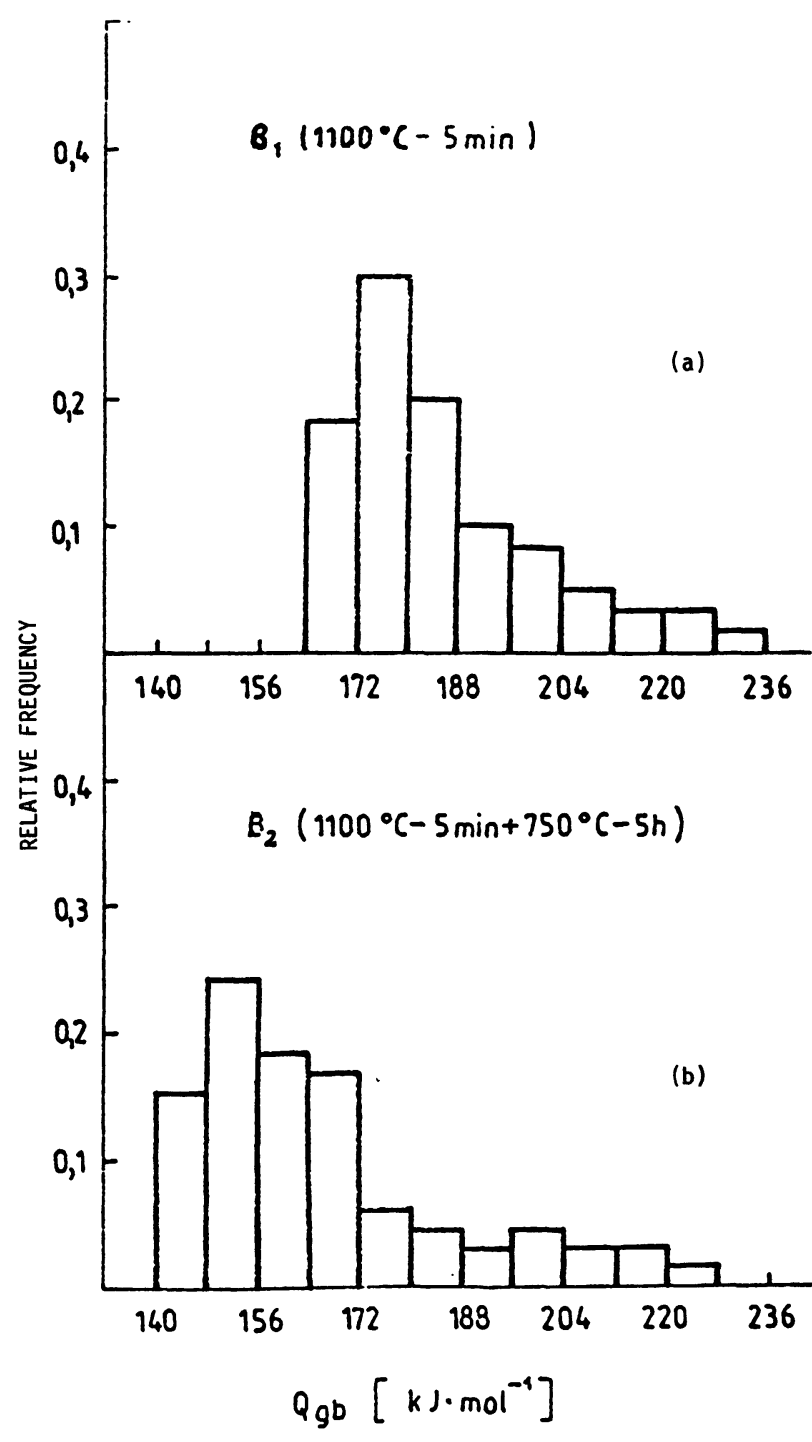

Fig. 18. - Histograms of grain boundary diffusion activation energies in high carbon austenitic steels [70]. a) after quenching from $1100^{\circ} \mathrm{C}$; b) after furnace cooling and ageing at $750^{\circ} \mathrm{C}$ : the carbide precipitation on grain boundaries involves a intergranular depletion in chromium and an increased diffusivity.

The second type of configuration has been observed in boundaries in both metals [65] and ceramics [40]. They give the boundaries a « hard » character, rendering them relatively resistant to sliding, leading to the formation of cavities at triple junctions, as has been observed in magnesia-doped alumina (Fig. 19). These considerations explain the observations made during studies on creep (cf. Sect. 3.1.3), and show that misorientation has an essential influence on the high temperature properties of grain boundaries.

The behaviour of extrinsic dislocations is strongly, influenced by intergranular segregation, due to several different reasons :

- the diffusivity distribution curve is displaced towards higher activation energies (Fig. 18) [70] ;
- dissociation can be obstructed : this effect has been analyzed theoretically [72] and has been observed in a boundary with $\Sigma=3$ in a sulphurized nickel foil [56];

- the dislocations can be pinned by solute atoms (Cottrell atmosphere type effect) $[65,73,74]$;

- segregation can modify the friction forces which oppose dislocation glide in the boundary [75, 76] : this effect could account for its effect on low temperature properties.

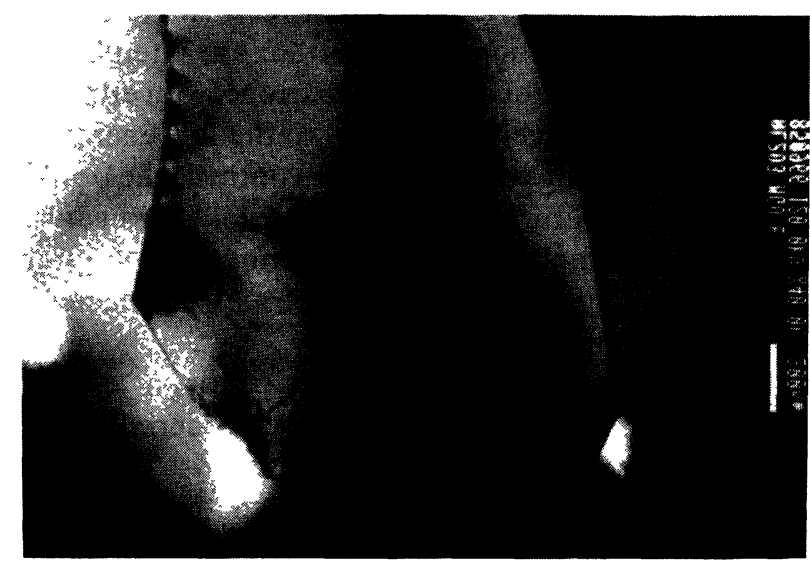

Fig. 19. - Bright field electron micrographs of a deformed sample of magnesia-doped alumina. Three boundaries contain extrinsic dislocation networks. Cavities (arrowed) appear at the edges of the special boundary $Z_{1} / Z_{4}$ [40].

3.2.4 Conclusions. - The apparently arbitrary distinction between intergranular properties, depending on whether the dominant influence is due to misorientation or to the orientation of the boundary plane, is justified by the analysis of both the processes involved and the kinetics of accommodation of extrinsic dislocations. While the misorientation alone is unable to explain the mechanical behaviour of a boundary, it determines its probability to be ductile or brittle, this being dependent also on the degree of segregation and the orientation of the boundary plane. On the contrary, the latter two interdependent factors control the intergranular corrosion resistance.

The response of a polycrystalline material to mechanical loading and to aggressive environments is an essential consideration for the majority of applications, so that it is clearly important to know the distribution of boundary orientations and misorientations, together with the factors which influence them. Since the long term objective is to be able to predict the contribution of grain boundaries to the overall properties, the last section of this review paper will be devoted to the question of grain boundary texture. 


\section{Grain boundary texture.}

The distributions of misorientations and boundary planes in the practical polycrystalline materials discussed in the present chapter must be clearly distinguished from those obtained in rotating crystallite experiments [77-79]. In the latter case, the grains are free to rotate, whereas in random polycrystals they are constrained and do not therefore adopt positions dictated solely by texture-related grain boundary energy minima. The aims of the two types of investigation are thus quite different. Determinations of the misorientation between single crystal spheres and a surrounding substrate are intended to relate the high frequency of occurrence of certain $\Sigma$ values, and/or of certain planes, to the low energy of the corresponding boundaries. In contrast, the objective of the experiments described below is to gain a better understanding of the contribution of grain boundaries to the functional properties of engineering polycrystals.

Grain boundary texture will depend on a large number of factors, which can be considered under two major headings :

* material-related parameters ; nature (metal, ceramic), crystallographic system, chemical composition ;

* parameters governed by the polycrystal preparation technique ; fabrication mode (solidification, recrystallization, sintering), temperature and duration of heat treatments (particularly important as regards grain size), cooling rate (which can have a marked influence on segregation phenomena), uniaxial or isostatic pressure (in the case of sintering).

4.1 Distribution OF MISORIENTATIONS. - Probability calculations for an f.c.c. polycrystal with randomly distributed grain orientations [25] give :

$9 \%$ CSL boundaries $\left(\Sigma \leqslant 25\right.$ and $\Delta \theta_{c}$ as defined by Brandon [24]).

$60 \%$ CAD boundaries (with $\langle 111\rangle,\langle 100\rangle$ and $\langle 110\rangle$ misorientation axes).

However, real distributions, which are necessarily related to the material texture, are always found to deviate from these predictions. Experimental results show that the percentage of CSL boundaries is often higher than that associated with a random distribution of grain orientations. On the contrary, the « theoretical » proportion of CAD boundaries tends rather to overestimate their effective frequency. This situation raises the fundamental question of whether the existence of special boundaries is the cause or the consequence of the overall texture.

Only a very few systematic investigations have been devoted to this area, and analysis of the results proves extremely difficult, since the distribution of grain boundary characteristics does not appear to be related to the material microstructure in a straightforward manner. The percentage of geomet- rically special boundaries varies with the thermomechanical history of the polycrystal, and depends particularly on the stage at which boundary formation occurs, and on the point where interstitial or substitutional elements begin to segregate. Nevertheless, in spite of the diversity of the published observations, a few common tendencies can be discerned, and these will be treated below.

4.1.1 Influence of boundary misorientation on the size and shape of the grains. - The percentage of special boundaries in a given material decreases when the grain size increases.

This effect has been observed for an $\mathrm{Fe}-3 \% \mathrm{Si}$ alloy, with grains larger than $5 \mu \mathrm{m}$, in which the misorientations were analyzed by the ECP (electron channeling pattern) technique [80]. It can also be implied from the observation (cf. Sect. 3.2.3) of the frequency of occurrence of boundaries containing extrinsic dislocations in aluminium subjected to abnormal grain growth, during which special boundaries are eliminated and replaced by more mobile general boundaries [81, 82]. These observations are in agreement with predictions based on a simple model involving the growth of a regular array of polygonal grains [83].

However, this relationship has not been confirmed when the increase in grain size is the result of normal growth. Moreover, the comparison of different materials is virtually impossible due to the overriding influence of alloying elements, even in trace amounts, on recrystallization phenomena. Thus, after identical thermomechanical treatments, pure iron was found to show a coarse grain size and a large proportion of special boundaries $(50 \%)$, whereas several iron alloys had fine-grained structures with only $25 \%$ of special boundaries [84] Furthermore, these proportions varied depending on whether the alloying elements were in solid solution or in the form of precipitates.

Finally, it should be noted that, in addition to this grain size effect, grain shape can also be important. In effect, in a nickel-base superalloy, boundary distributions in regions with a banded structure were found to be different from those in areas with equiaxed grains [85].

\subsubsection{Influence of polycrystal preparation on bound-} ary misorientation. - The distribution of boundary orientations after secondary recrystallization depends strongly on that existing after primary recrystallization [86]. In the extreme case, it can be easily understood that the distribution of grain boundary types will be completely different in a polycrystal obtained by annealing a deformed single crystal than in one produced from an initially polycrystalline state.

In the first case, the distribution of boundaries depends on the initial orientation of the single 
crystal and on the amount of strain prior to annealing. The percentage of boundaries with misorientations close to coincidence positions $(\Sigma \leqslant 29)$ increases with prior strain whereas the proportion of low angle boundaries decreases [7].

The method of polycrystal preparation does not appear to affect the possibility of formation of CSL boundaries. However, the frequency of low angle boundaries has been found to be lower (3\%) in polycrystalline iron sintered at $700^{\circ} \mathrm{C}$ than in iron rolled and annealed at the same temperature $(20 \%)$ [86].

A certain number of observations in apparent contradiction with the above relationships can probably be explained by the fact that purity has not been considered explicitly, whereas this is certainly one of the most important parameters.

4.1.3 Influence of purity on boundary misorientation. - It is important to emphasize from the outset that grain boundary characteristics are affected not so much by the total impurity content, as by the distribution of particular elements, even when present in trace amounts, and by their chemical state (in atomic or combined form).

The frequency of CSL boundaries in zone-refined high purity aluminium has been found to be strongly affected by very small amounts of tin. Thus, only $10 \mathrm{ppm}$ Sn was sufficient to increase their proportion from 20 to $50 \%$, whereas no effect was observed for titanium additions [87]. Unfortunately, no details were given in this work on the localization of the tin atoms.

The analysis of pure nickel polycrystals containing traces of sulphur shows that the total content of this element has little influence on the distribution of special $\left(\Sigma \leqslant 19, \Delta \theta \leqslant \Delta \theta_{c}\right.$ Brandon) and general boundaries, in so far as intergranular segregation is not very probable during the grain formation process (compare (a) and (b) in Fig. 20) [88]. Subsequent

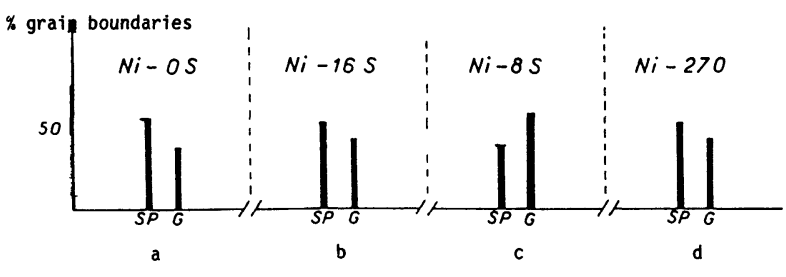

Fig. 20. - Histograms showing the frequencies of special boundaries (CSL and LA) in nickel polycristals in function of their sulphur content depending on the matrix content and on the thermal treatments [88]. a) pure nickel recrystallized at $1050^{\circ} \mathrm{C}$ during 4 hours; b) $\mathrm{Ni}$ $16 \times 10^{-6} \mathrm{P}$ - recrystallization as previously followed by segregation at $625^{\circ} \mathrm{C}, 2$ hours ; c) Ni- $8 \times 10^{-6} \mathrm{P}$ - simultaneous recrystallization and segregation at $625^{\circ} \mathrm{C}$, 12 days ; d) $\mathrm{Ni}-270$ analyzed by Laue technique [89] - to be compare to $b$ ). segregation is unable to modify the misorientations (cf. Sect. 3.2.1). However, when the special boundaries are considered in more detail, by separating them into twins $(\Sigma=3)$ and other coincident orientations, certain differences appear, related solely to purity (Fig. 21a and b). The absence of low angle boundaries and the large proportion of twins is probably due to the high recrystallization temperature $(5)$.

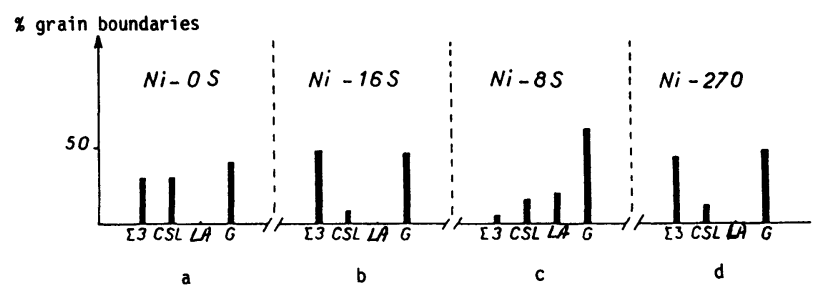

Fig. 21. - Idem figure 20 with details of the special boundary distributions.

When the recrystallization and segregation phenomena occur simultaneously, the frequency of general boundaries increases sharply, the proportions of the two types being reversed (Fig. 20c).

Similar resultats were obtained in the iron-phosphorus system. Figure 22 shows the cumulative fre-

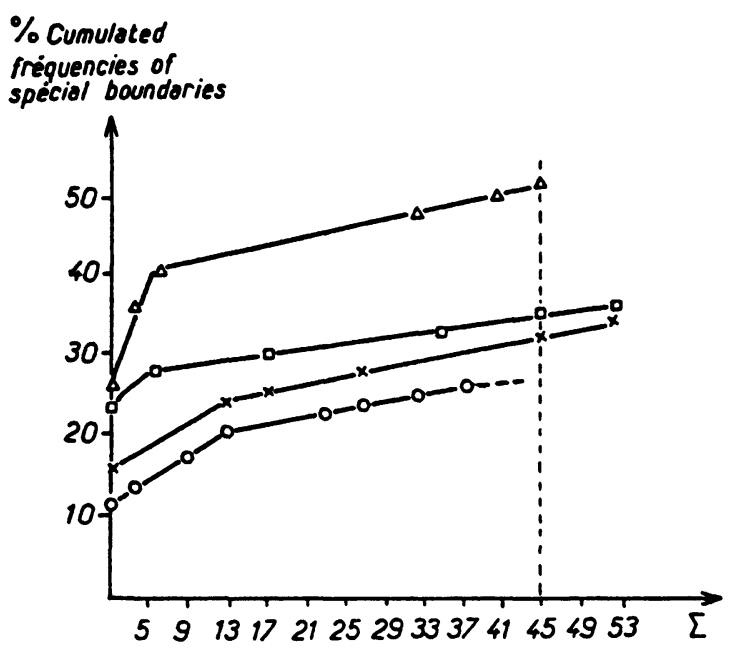

Fig. 22. - Cumulated frequencies of special boundaries in various $\mathrm{Fe}-\mathrm{P}$ specimens $69: \mathrm{Fe}-40 \times 10^{-6} \mathrm{P}$ annealed at $600^{\circ} \mathrm{C}(\times)$ or at $720^{\circ} \mathrm{C}(\triangle), \mathrm{Fe}-100 \times 10^{-6} \mathrm{P}$ annealed at $600^{\circ} \mathrm{C}(\mathrm{O})$ or at $720^{\circ} \mathrm{C}(\square)$. Strong segregation of Phosphorus in grain boundaries is well known to occur at $600{ }^{\circ} \mathrm{C}$ while a low segregation level is expected at $720^{\circ} \mathrm{C}$ [69].

$\left({ }^{5}\right)$ These distributions, established for a relatively limited number of boundaries analyzed by TEM, were confirmed by Laue diffraction experiments on 222 boundaries, leading to a histogram perfectly identical to that obtained for the Ni-16 S material (Figs. 20, 21d) [89]. 
quency diagram for near-coincidence boundaries of increasing $\Sigma$ ( $\Sigma \leqslant 45$ and $\Delta \theta=\Delta \theta_{c}$ Brandon), for four iron samples with different total contents and distributions of phosphorus. Only the specimen whose boundaries are relatively unlikely to contain phosphorus show a significantly higher proportion of special orientations [69].

In contrast, the presence of additive elements such as magnesium and yttrium in sintered alumina increases the percentage of special boundaries (Fig. 23) [90] ; the fact that these elements act as grain growth inhibitors suggests that they segregate to boundaries. However, it must be emphasized that the grain size in these doped aluminas is extremely fine $(0.5 \mu \mathrm{m})$, compared to those of pure alumina (10 to $50 \mu \mathrm{m})$, and that this size effect can also influence the distribution of boundaries (cf. Sect. 4.1.1).
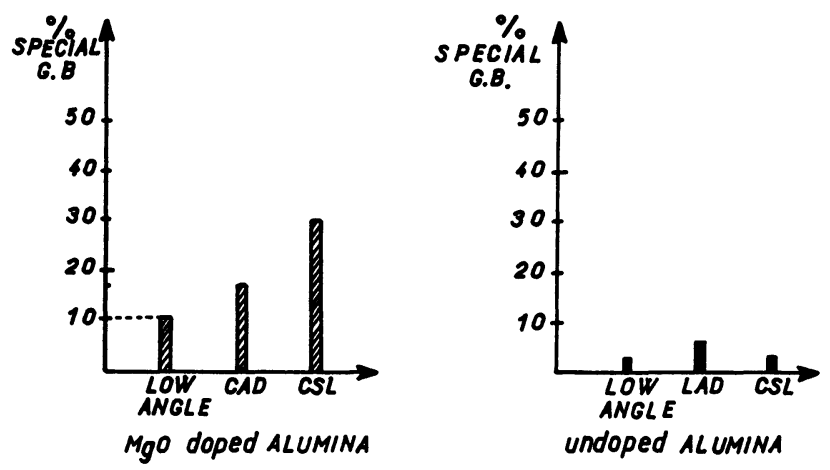

Fig. 23. - Distribution of special grain boundary misorientations in the $\mathrm{Mg}$-doped and undoped aluminas [90].

In conclusion, these initial investigations on the distribution of misorientations demonstrated that it is difficult to specify the respective influences of the various factors related either to the material or to its mode of fabrication. With the present improved understanding of grain textures, it can now be attempted to establish a relationship between the overall texture and the misorientations between grains. However, any function describing the grain orientation distribution will necessarily have a macroscopic nature and cannot therefore take account of the near-neighbour relations characteristic of grain boundary texture. One approach to this problem is to determine local textures.

\subsection{THE DISTRIBUTION OF BOUNDARY PLANES. -} Published investigations on boundary plane distributions are even less numerous than in the case of misorientations, since statistical techniques either cannot be used (ECP), or the associated misorientations are unknown (Auger spectroscopy). Transmission electron microscope analysis requires the study of a sufficient number of grain boundaries to be statistically significant. It is probably safe to assume that there is little tendency for boundary planes to readjust in polycrystalline thin foils.

In alumina, the occurrence of «special » orientations parallel to close packed lattice planes, particularly the basal plane (0001), seems to be favoured by the purity of the ceramic material (Fig. 24) [90].
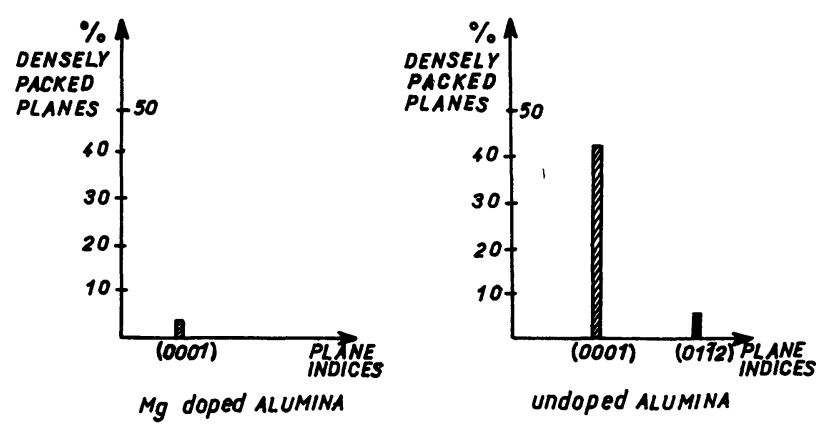

Fig. 24. - Distribution of special grain boundary planes in the $\mathrm{Mg}$-doped and undoped aluminas [90].

However, the purity does not explain alone this selection. In a fine grain $\mathrm{MgO}$-doped alumina, highly deformed by compression at $1500^{\circ} \mathrm{C}$, grain growth has occured in some regions of the sample ; in that case, a large number of grain boundary planes, either straight or faceted, correspond to a dense plane in one crystal at least. Thus, selection of dense planes and faceting may be a result of the growth processes [91]. This remark does not rule out the propensity for grain boundary free of solute to preferentially migrate. In alumina special grain boundary misorientation and special grain boundary plane orientation appear mutually exclusive [91].

In the case of iron containing from 40 to $100 \mathrm{ppm}$ phosphorus, the boundary planes are never parallel to the closest packed planes $\{110\}$ of the bcc structure [69]. These observations confer a certain reciprocal character to the relationship between grain boundary plane and segregation (cf. Sect. 3.2.1).

In contrast with the grain boundary misorientation distribution, no relationship may be theoretically envisaged between the grain boundary plane distribution and any other microstructural characteristic ; only empirical relation might be found. Thus properties controlled by the grain boundary plane orientation appear unlikely to predict.

\section{Summary and conclusion.}

Although no absolute geometrical criterion of intergranular speciality may be defined, grain boundaries behave differently according to their geometry, some of them being «special » in practice. Looking 
at the existing results, a distinction can be made between intergranular properties depending mainly on the grain boundary misorientation, essentially mechanical properties, and those most directly related to the grain boundary plane orientation such as intergranular diffusion. Concerning the first type of properties, grain boundaries which distinguish themselves from others are near three dimensional or near one-dimensional coincidence boundaries. Most results about the second type of properties single out boundaries with a high atomic planar density rather than a high coincidence site density.

The possibility to obtain a specific polycristalline property via the control of grain boundaries must differ according to the predominant influence of one or the other cristallographical parameter on the intergranular processes involved. In so far as the grain boundary misorientation distribution is necessarily linked to the grain texture, despite the fact that until now no straightforward relationship has been established between them, it is suggested that a misorientation control might be obtained. On the contrary, any attempt to get a controlled distribution of the grain boundary plane orientations is hardly conceivable.

Finally, coming back to the "Grain Boundary Design " concept, prospects for tailoring mechanical properties of polycristalline materials via the control of grain boundary misorientation distributions appear feasible. With this aim in view, the influence of segregation phenomena have to be considered, not only they may modify the ductile or brittle character of grain boundaries but they are also able to change the grain boundary texture.

\section{References}

[1] Balluffi R. W., Metall. Trans. A 13 (1982) 2069.

[2] Sutton A. P., Int. Met. Rev. 29 (1984) 377.

[3] Vitek V., Sutton A. P., Smith D. A., Pond R. C., Grain Boundary Structure and kinetics, Ed. by R. W. Baluffi, Am. Soc. Met., Metals Park Ohio (1980) p. 115.

[4] Vitek V., Wang G. J., J. Phys. Colloq. France 43 (1982) C6-147.

[5] Vitek V., Minonishi Y., Wang G. J., J. Phys. Colloq. France 46 (1986) C4-171.

[6] OH Y., ViteK V., Acta Metall. 34 (1986) 1941.

[7] Watanabe T., Res. Mechanica 11 (1984) 47.

[8] Rosenhaim W., Humphrey J. C. W., J. Iron Steel Inst. 87 (1913) 319.

[9] Read W. T., Schockley W., Phys. Rev. 78 (1950) 275.

[10] Friedel G., Leçons de Cristallographie, Ed. Blanchard (1926).

[11] BollmanN W., Crystals Defects and Crystalline Interfaces (Springler Verlag N.Y., Berlin) 1970.

[12] Schober T., Balluffi R. W., Philos. Mag. 20 (1969) 511.

[13] Schober T., Balluffi R. W., Philos. Mag. 21 (1970) 109.

[14] Bollmann W., Michaut B., Sainfort G., Phys. Status Solidi A 13 (1972) 637.

[15] Balluffi R. W., Sass S. L., Schober T., Philos. Mag. 26 (1972) 585.

[16] Sass S. L., TaN T. Y., Balluffi R. W., Philos. Mag. 31 (1975) 559.

[17] Pumphrey P. H., Scri. Metall. 6 (1972) 107.

[18] Warrington D. H., Grimmer H., Philos. Mag. 30 (1974) 461.

[19] Schindler R., Clemans J. E., Balluffi R. W., Phys. Status Solidi A 26 (1979) 749.

[20] Bonnet R., Cousineau E., Acta Crystallogr. A 33 (1977) 850.

[21] Bleris G. L., Nouet G., Hagege S., DelavigNeTte P., Acta Crystallogr. A 38 (1982) 550.
[22] Chen F. R., King A. H., Philos. Mag. A 57 (1988) 431.

[23] Grimmer H., Bonnet R., Lartigue S., Priester L., à paraître.

[24] Brandon D. G., Acta Metall. 14 (1966) 1479.

[25] Warkington D. H., Boon M., Acta Metall. 23 (1975) 599.

[26] Ishida Y., MC Lean M., Philos Mag. 27 (1973) 1125.

[27] Crocker A. G., Doguenan M., Ingle K. W., Philos. Mag. A 41 (1980) 21.

[28] Pond R. C., Vitek V., Smith D. A., Acta Metall. 27 (1979) 235.

[29] Sutton A. P., Vitek V., Philos. Trans. R. Soc. London A 309 (1983) 1, 37, 55.

[30] Wang G. J., Sutton A. P., Vitek V., Acta Metall 32 (1984) 1093.

[31] Balluffi R. W., Bristowe P. D., Surf. Sci. 144 (1984) 28.

[32] Bristowe P. D., Balluffi R. W., J. Phys. Colloque France 46 (1985) C4-155.

[33] Kwan E. P., Balluffi R. W., Philos. Mag. A 56 (1987) 137.

[34] Wolf D., J. Phys. Colloq. France 46 (1985) C4-197.

[35] Paidar V., Phys. Status. Solidi A 92 (1985) 115.

[36] Paidar V., Acta Metall. 35 (1987) 2035.

[37] Sutton A. P., Balluffi R. W., Acta Metall. 35 (1987) 2177.

[38] Watanabe T., Shima S., Karashima S., Proc. AIME Symposium on liquid and solid embrittlement. Ed. M. H. Kamdar (1982).

[39] Watanabe T., Tanaka M., Karashima S., Idem [38], p. 38.

[40] Lartigue S., Priester L., Proc. JIMIS 4, Grain boundary structure and related phenomena, Trans. JIM 27 (1986) p. 205.

[41] LiM L. C., RAJ R., Acta Metall. 32 (1984) 1183.

[42] Lagarde P., Biscondi M., Can Metall. Q. 13 (1984) 245. 
[43] Michaut B., Silvent A., Sainfort G., Mem. Sci. Rev. Metal. 71 (1974) 527.

[44] Lagarde P., Biscondi M., J. Phys. Colloq. France 36 (1975) C4-297.

[45] Hokawa H., Watanabe T., Karashima S., Philos. Mag. A 44 (1981) 1239.

[46] Horton C. A. P., Silcock J. M., J. Microsc. 102 (1974) 339.

[47] SiCKAFUS K., SASS S. L., Scr. Metall. 18 (1984) 165.

[48] Pond R. C., Vitek V., SMith D. A., Acta Crystallogr. A 35 (1979) 689.

[49] Messmer C. P., Briant C. L., Acta Metall. 30 (1982) 457.

[50] Hashimoto M., Ishida Y., WaKayada S., Yamamoto R., Doyama M., Acta Metall. 32 (1984) 1-21.

[51] LaRere A., Masuda-Jindo K., Yamamoto R., Doyama M., Proc. JIMIS 4, Grain boundary and related phenomena, Trans JIM 27 (1986) p. 225.

[52] Susuki S., Akibo K., Mikura K., Scr. Metall. 15 (1981) 1139.

[53] Ogura T., Mc Mahon C. J., Feng H. C., Vitek V., Acta Metall. 26 (1978) 1317.

[54] Bouchet D., Priester L., Scr. Metall. 20 (1986) 961.

[55] Bouchet D., Priester L., Scr. Metall. 21 (1987) 475.

[56] Bouchet D., Auffray B., Priester L., J. Phys. France Colloq. 49 (1988) C5-417.

[57] Robinson J. T., Peterson N. C., Acta Metall. 16 (1973) 1181.

[58] Herbeuval I., Biscondi M., Can. Metall. Quart. 13 (1974) 171.

[59] Peterson N. L., Int. Met. Rev. 28 (1983) 64.

[60] BISCONDI M., Physical Chemistry of the solid state : Applications to metals and their compounds. Ed. by P. Lacombe. (Elsevier Pub.) 1984, p. 225.

[61] Assassa W., Guiraldenc P., Beaunier L., FroMENT M., J. Phys. Colloq. France 36 (1975) C4225.

[62] LeMUET D., Thèse (Grenoble) 1981.

[63] Balluffi R. W., Metall. Trans. 13A (1982) 2069.

[64] Valiev R. Z., Gertsman V. YU., KaIbysheV O. A., Phys. Status Solidi A 61 (1980) K95.

[65] Lartigue S., Priester L., Acta Metall. 31 (1983) 1809.

[66] Priester L., Les joints de grains dans les matériaux (Ed. de Physique) 1985, p. 231.

[67] Grabski M. W., Matér. Tech., n 8-9 (1985) E97.
[68] Johanneson T., Thölen A., Metal. Sci. J. 16 (1972) 189.

[69] El M'rabat B., Priester L., Mat. Sci. Eng. A 101 (1988) 117.

[70] Swiatnicki W., Grabski M. W., Acta Metall. 34 (1986) 817.

[71] SWIATNICKI W. A., LOJKOWSKI W., GRABSKI M. W., Acta Metall. 34 (1986) 599.

[72] IshidA Y., MORI M., J. Phys. Colloq. France 46 (1985) C4-46.

[73] Jonas A. R., Howell P. R., Ralph B., J. Met. Sci. 11 (1976) 1593 (1) 1600 (2).

[74] Lartigue S., Bouchet D., Priester L., Physical chemistry of the solid state: Applications to metals and their compounds, Ed. P. Lacombe (Elsevier Pub.) 1983, p. 183.

[75] Biscondi M., J. Phys. Colloq. France 43 (1982) C5293.

[76] Schulson E. M., Weihs T. P., BaKer J., Frost H. J., Horton J. A., Acta Metall. 34 (1986) 1395.

[77] Chaudari P., Matthews J. W., J. Appl. Phys. 42 (1971) 3063.

[78] Herrmann G., Gleiter H., Baro G., Acta Metall. 24 (1976) 357.

[79] Mykura H., Bansal P. S., Lewis M. H., Philos. Mag. 42A (1980) 225.

[80] Watanabe T., Kawamata Y., Karashima S., Proc. JIMIS 4 ; Grain boundary and related phenomena, Trans JIM 27 (1986) p. 601.

[81] Grabski M. W., J. Phys. Colloq. France 46 (1985) C4-567.

[82] Wyrzykowski J. W., Grabski M. W., Philos. Mag. A 53 (1986) 505.

[83] Watanabe T., Scr. Metall. 2 (1987) 427.

[84] Lartigue S., Khalfallah O., Priester L., Rev. Phys. Appl. 17 (1982) 649.

[85] Randle V., RalPh B., Dingley M., Acta Metall. 36 (1988) 267.

[86] Harase J., Shimizu R., Kuroki K., Nakayama T., Wada T., Watanabe T., Proc. JIMIS 4, Grain boundary and related phenomena, Trans JIM 27 (1986) p. 563.

[87] Belluz R. W., Aust K. T., Met. Trans. 6A (1975) 219.

[88] Beaunier L., Bouchet D., Colliex C., Trebbia P., Vignaud C., Rapp. ATP, Surfaces et interfaces (1984).

[89] Lim L. C., RAJ R., Acta Metall. 32 (1984) 1171.

[90] Lartigue S., Priester L., J. Phys. France Colloq. 49 (1988) C5-451.

[91] Lartigue S., Thèse (Orsay) 1988. 